\title{
Validation of a numerical model with a benchmark experiment for melting governed by natural convection in latent thermal energy storage
}

\author{
Julian Vogel ${ }^{*}$, André Thess ${ }^{* * * *}$ \\ * Institute of Engineering Thermodynamics, German Aerospace Center (DLR), Pfaffenwaldring 38-40, 70569 Stuttgart, Germany \\ ** University of Stuttgart, Pfaffenwaldring 31, 70569 Stuttgart, Germany
}

J. Vogel, A. Thess, Validation of a numerical model with a benchmark experiment for melting governed by natural convection in latent thermal energy storage, Appl. Therm. Eng. 148 (2019) 147-159.

https://doi.org/10.1016/j.applthermaleng.2018.11.032.

\begin{abstract}
We report a combined experimental and numerical investigation of a melting process representative of latent thermal energy storage systems. The purpose of the work is to assess the accuracy of numerical models of melting governed by natural convection with a benchmark experiment. The experiment consists of a rectangular box filled with a model liquid (n-octadecane) and heated symmetrically from both sides such as to allow access for shadowgraph imaging and particle image velocimetry to measure the phase state and velocities, respectively. Our numerical method for computing fluid flow, temperature, and phase state involves two different approaches: the first is a detailed model using variable thermophysical properties and the volume of fluid method to allow volume expansion in an additional air phase that we solve in two dimensions. The second is a simplified model using constant thermophysical properties and the Boussinesq approximation that we solve either in two or in three dimensions. In the first part of the work, we systematically compare the simplified (Boussinesq) with the detailed (volume of fluid) model. We find that for the given set of parameters $\left(\mathrm{Ra}=2 \cdot 10^{8}, \mathrm{~A}=4, \mathrm{Ste}=0.092, \mathrm{Pr}=52\right)$, the difference between the detailed and the simplified model in predicting global quantities such as the liquid phase fraction and the total heat flow rate is smaller than 4 percent, whereas velocities differ up to 20 percent. In the second part of the work, we compare the simulations of the simplified Boussinesq model in three dimensions with the benchmark experiment. We find that the simulation predicts the liquid phase fraction and temperatures with deviations below 4 percent, but significantly overestimates the velocity magnitudes. Our experimental and numerical tools provide a rational framework in which the accuracy of latent thermal energy storage simulations can be systematically and comprehensively assessed.
\end{abstract}

\section{Keywords}

Phase change material (PCM); Solid-liquid phase change; Computational Fluid Dynamics (CFD); Boussinesq approximation; Volume of Fluid method (VOF).

\footnotetext{
* Corresponding author E-mail address: julian.vogel@dlr.de, ORCID ID: https://orcid.org/0000-0001-9792-2332
} 


\section{Nomenclature}

Latin

A aspect ratio

a thermal diffusivity, $[a]=\mathrm{m}^{2} / \mathrm{s}$

$B$ momentum source coefficient, $[B]=(\mathrm{Pa} \mathrm{s}) / \mathrm{m}^{2}$

$C \quad$ mushy zone constant, $[C]=(\mathrm{Pa} \mathrm{s}) / \mathrm{m}^{2}$

$c_{p} \quad$ specific isobaric heat capacity, $\left[c_{p}\right]=\mathrm{J} /(\mathrm{kg} \mathrm{K})$

$D \quad$ depth, $[D]=\mathrm{m}$

$\hat{\boldsymbol{e}}_{y} \quad$ unit vector in $y$-direction

$\boldsymbol{F}_{b} \quad$ buoyancy term, $\left[\boldsymbol{F}_{b}\right]=\mathrm{Pa} / \mathrm{m}$

Fo Fourier number

$f_{i} \quad$ linear interpolation function

$f_{\mathrm{l}} \quad$ liquid phase fraction

$g$ gravity constant, $[g]=\mathrm{m} / \mathrm{s}^{2}$

$H \quad$ height, $[H]=\mathrm{m}$

$h \quad$ specific enthalpy, $[h]=\mathrm{J} / \mathrm{kg}$

I unit tensor

$k \quad$ thermal conductivity, $[k]=\mathrm{W} /(\mathrm{m} \mathrm{K})$

$\dot{m} \quad$ volumetric mass flow, $[\dot{m}]=\mathrm{kg} /\left(\mathrm{m}^{3} \mathrm{~s}\right)$

$p \quad$ pressure, $[p]=\mathrm{Pa}$

$\dot{Q} \quad$ heat flow rate, $[\dot{Q}]=\mathrm{W}$

$q \quad$ constant in momentum source coefficient $B$

$L \quad$ latent heat, $[L]=\mathrm{kJ} / \mathrm{kg}$

$R a_{H} \quad$ Rayleigh number

$S_{h} \quad$ source term in energy equation, $\left[S_{h}\right]=\mathrm{W} / \mathrm{m}^{3}$

$\boldsymbol{S}_{\boldsymbol{u}} \quad$ source term in momentum equation, $\left[\boldsymbol{S}_{\boldsymbol{u}}\right]=\mathrm{Pa} / \mathrm{m}$

Ste Stefan number

$T$ temperature, $[T]={ }^{\circ} \mathrm{C}$

$t \quad$ time, $[t]=\mathrm{s}$

$\operatorname{Pr} \quad$ Prandtl number

$\boldsymbol{u} \quad$ velocity vector, $\boldsymbol{u}=(u, v, w)^{\top}$

$u \quad x$-velocity, $[u]=\mathrm{m} / \mathrm{s}$

$v \quad y$-velocity, $[v]=\mathrm{m} / \mathrm{s}$

$w \quad z$-velocity, $[w]=\mathrm{m} / \mathrm{s}$
$\begin{array}{ll}W & \text { width, }[W]=\mathrm{m} \\ x, y, z & \text { coordinates, }[x, y, z]=\mathrm{m}\end{array}$

Greek

$\alpha \quad$ volume fraction in VOF model

$\beta \quad$ thermal expansion coefficient, $[\beta]=1 / \mathrm{K}$

$\delta \quad$ distance of free surface from max. height, $[\delta]=\mathrm{m}$

$\mu \quad$ dynamic viscosity, $[\mu]=$ Pa s

$v \quad$ kinematic viscosity, $[v]=\mathrm{m}^{2} / \mathrm{s}$

$\xi \quad$ half melting range in melting point model, $[\xi]={ }^{\circ} \mathrm{C}$

$\rho \quad$ density, $[\rho]=\mathrm{kg} / \mathrm{m}^{3}$

$\boldsymbol{\tau} \quad$ stress tensor, $[\boldsymbol{\tau}]=\mathrm{Pa}$

\section{Subscripts}

$0 \quad$ initial value

cond conduction

conv convection

exp experimentally measured values

1 liquid

lat latent heat content

m melting point

max maximum

min minimum

rad radiation

ref reference

s solid

sens sensible heat content

w wall

Symbols

$\nabla \quad$ nabla operator: $\nabla=(\partial / \partial x, \partial / \partial y, \partial / \partial z)$

$\Delta \quad$ finite difference

\section{Introduction}

Latent thermal energy storage (LTES) systems represent an important building block of future energy systems that bridge the gap between fluctuating supply of renewable energy and heat demand. An overview of LTES is given by Mehling and Cabeza [1] and Bauer et al. [2]. Although LTES has been widely investigated in the past both experimentally and numerically, a systematic analysis and experimental validation of the full set of space- and time-resolved phase boundary shapes and velocity fields due to natural convection during melting in a typical LTES geometry has never been carried out.

The first objective of the present work is to analyze different modeling approaches by comparing a detailed numerical model with a simplified numerical model. The detailed model uses temperature-dependent thermophysical properties and allows a variable density (and thus volume expansion during change of phase and temperature) with the Volume of fluid method (VOF). The simplified model uses constant properties and incorporates buoyancy through the Boussinesq approximation.

The second objective of this work is to validate a simulation model using the high-accuracy benchmark experiment by Vogel and Bauer [3]. A thorough comparison of the space- and time-resolved liquid phase fraction and velocities in the liquid phase, as well as time-resolved temperatures at distinct points, clearly identifies the accuracy of the modeling approach.

\section{State of research}

Numerical models for natural convection dominated melting have been investigated for more than thirty years, one of the early works being by Voller et al [4]. Most of the existing models were collected in the reviews by Salcudean and Abdullah[5], Samarskii et al. [6], Voller [7], $\mathrm{Hu}$ and Argyropoulos [8], Dutil et al. [9] and Dhaidan et al. [10]. The models were roughly divided in deforming grid and fixed grid methods. A comparison of these approaches was done by Lacroix and Voller [11], and Viswanath and Jaluria [12]. The 
variables have been either formulated in primitive variables, such as pressure and velocity, or in a stream function and vorticity approach [6]. In the fixed grid methods with primitive variables, the enthalpy-porosity technique by Voller and Prakash [13], Brent et al. [14] or Voller and Swaminathan [15] has often been used for phase change and the incompressible Navier-Stokes equations have been solved with a projection method to decouple the computation of pressure and velocity. Semi-implicit projection methods such as the SIMPLE algorithm [16] have been used by most researchers, but also an explicit projection method [17] has been applied by Galione et al. [18] and Kozak and Ziskind [19]. Mostly, the Boussinesq approximation has been applied, which sets a constant density in all terms but the buoyancy term, where the dependency of density with temperature is linearized. A variable PCM density was implemented in conjunction with an air phase and the volume of fluid (VOF) method [20] to model volume expansion by Shatikian et al. [21], Shmueli et al. [22] and Hosseinizadeh et al. [23]. However, the impact of variable material properties and volume expansion on melting governed by natural convection is not known, since the results of a detailed VOF approach and a simplified Boussinesq approximation have not yet been compared.

Despite the impressive amount and level of sophistication of these numerical models, their verification and validation are still remaining issues. Different methods have been producing deviating results [24] and the agreement of numerical results with experiments has often not been satisfactory [25]. To give an example on the issue of verification: Bertrand et al. [26] and Gobin and Le Quéré [27] performed a thorough comparison of multiple well-designed models on a numerical benchmark test case. Although results indicated the same trends, the phase boundary differed up to $\pm 20 \%$ in one of the test cases. To give another example on the issue of validation: Campbell and Koster [28] repeated the most popular validation experiment, which was performed by Gau and Viskanta [29], with an improved experimental setup. Although the phase front shapes of the simulation and experiment were similar in the final steady state, they deviated during the temporal evolution.

Conclusively, verification and validation have been a challenging task. The problem is that there is a general lack of experiments for melting governed by natural convection suitable for validation [25]. For the geometry and boundary conditions found in latent thermal energy storage, hardly any specific validation experiment is available. Therefore, a novel validation experiment was designed by Vogel and Bauer [3]: a phase change material (PCM) with high Prandtl number was melted symmetrically from two vertical sides in a rectangular enclosure. Phase state and velocities were measured with high temporal and spatial resolution and temperatures were measured with high temporal resolution at distinct points to provide detailed data for validation.

\section{Benchmark test case}

The generic benchmark test case is illustrated in Figure 1: a PCM is isothermally heated from two opposing vertical sides in a rectangular enclosure of height $H$ and half width $W$. The bottom side is an adiabatic wall and the top side is an adiabatic free surface. The PCM is assumed to be initially solid at a temperature $T_{0}$ slightly below the melting point $T_{\mathrm{m}}$ of the PCM. At time $t_{0}$ the temperature at the heated walls $T_{\mathrm{w}}$ is raised to a temperature $T_{\mathrm{m}}+\Delta T$ and melting starts when the melting point is reached.

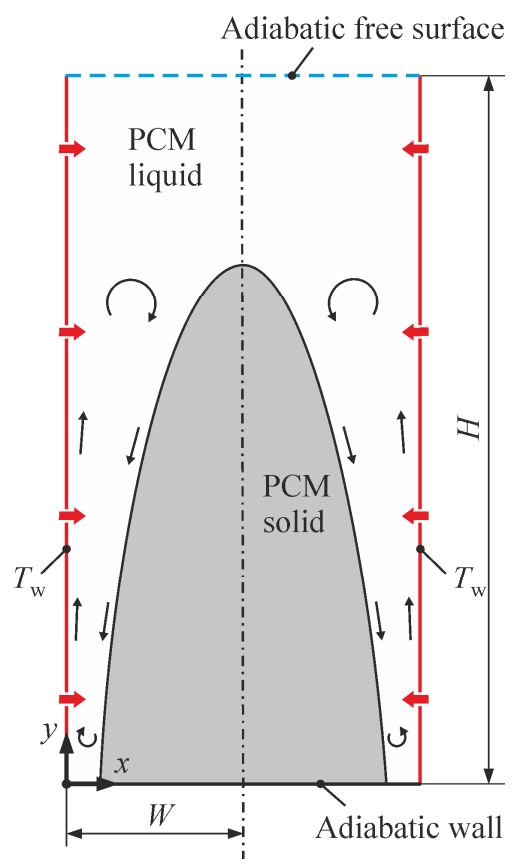

Figure 1: Benchmark test case of a PCM that is isothermally heated from two opposing vertical sides in a rectangular enclosure.

The relevant dimensionless numbers of the described physical problem are the Rayleigh number $R a_{H}$, the Stefan number Ste, the time dependent Fourier number $\mathrm{Fo}_{H}(t)$, the Prandtl number $\operatorname{Pr}$ and the Aspect ratio $A$ :

$$
\begin{aligned}
& R a_{H}=\frac{g \beta \Delta T H^{3}}{v_{1} a_{1}}, \quad \text { Ste }=\frac{c_{p, 1} \Delta T}{L}, \\
& F o_{H}(t)=a_{1} \frac{t}{H^{2}}, \quad \operatorname{Pr}=\frac{v_{1}}{a_{1}}, \quad A=\frac{H}{W} .
\end{aligned}
$$

The driving temperature difference is $\Delta T=\left(T_{\mathrm{w}}-T_{\mathrm{m}}\right)$ between the wall and the melting point of the PCM. The material properties are taken at the average liquid temperature $T_{\mathrm{m}}+\Delta T / 2$.

There is an ongoing discussion on whether the width $W$ or the height $H$ should be used as characteristic length. While research about natural convection in enclosures used the width, e.g. Elder [30], theoretical scaling analysis revealed the height as physically meaningful choice, e.g. Jany and Bejan [31]. In the end, the characteristic length may be freely chosen, as long as the aspect ratio $A$ of the enclosure is taken into account. In the present work, we chose the height $H$ as characteristic length.

Melting is initially dominated by heat conduction, but natural convection sets in as soon as the liquid layer reaches a critical size, so that the buoyancy forces due to temperature gradients can overcome the viscous forces due to boundary layers at the walls and at the liquid-solid interface. A criterion for the transition from the pure conduction regime to a natural convection affected regime for a fluid in a rectangular enclosure was derived by Batchelor [32]. The correlation is 
given here with the height used as characteristic length instead of the originally used width. The flow is then dominated by natural convection, if

$\frac{R a_{H}}{A^{4}} \geq 500$.

The onset of turbulence for a fluid in a rectangular enclosure was studied by Elder [33]. The correlation is also given with the height as characteristic length instead of the originally used width. The flow is characterized to be laminar for

$R a_{H}<10^{10}$.

Although these correlations were derived for a fluid without phase change, we can still apply them in the following approximate sense: the height and width of the liquid phase region will permanently change during melting. However, at a certain point, the liquid region will be nearly as high and/or wide as the enclosure height $H$ and width $W$. As a result, assuming the flow of a single phase fluid is governed by natural convection or turbulence. Then the flow in the liquid phase of a phase change material is probably also governed by natural convection or turbulence at some point during the melting process.

\section{Experimental method}

An experimental test bench for the benchmark test case described in the last section was built by Vogel and Bauer [3]. Detailed optical measurement techniques were used to highly resolve the phase state and velocities in the liquid phase. The phase state was measured with shadowgraphy and the velocities in the liquid phase were measured with particle image velocimetry (PIV). Temperatures were measured with thermocouples at three distinct positions in the PCM. In the following, the test bench design is described and material properties and experimental parameters are given.

\subsection{Test bench design and operation}

The experimental setup is shown in Figure 2: a transparent enclosure made of acrylic glass PLEXIGLAS $^{\circledR}$ contained heaters made of Steel 1.4301 and the PCM n-octadecane. The rest of the volume was filled with ambient air to allow volume change of the PCM during melting. The half width of the test section was $W=25 \mathrm{~mm}$. The height of the heaters and the initial fill height of the PCM in the liquid state were $H_{1}=$ $105 \mathrm{~mm}$. During solidification, the fill height of the PCM decreased by the length $\delta$ due to a larger density in the solid state. After solidification, $\delta$ was approximately $10 \mathrm{~mm}$ and the solid fill height was about $H_{\mathrm{s}}=95 \mathrm{~mm}$. The initial fill height in the liquid state was specifically chosen to achieve an average height of $H=100 \mathrm{~mm}$ during the melting process. The depth of the test volume $D=50 \mathrm{~mm}$ was large enough to neglect boundary effects at the midplane induced by the front and back walls. The initial temperature of the PCM was $1 \mathrm{~K}$ below the melting temperature: $T_{0}=27^{\circ} \mathrm{C}$. The wall temperature was set to $T_{\mathrm{w} \text {,middle }}=38{ }^{\circ} \mathrm{C}$, which resulted in a driving temperature difference $\Delta T=10 \mathrm{~K}$.
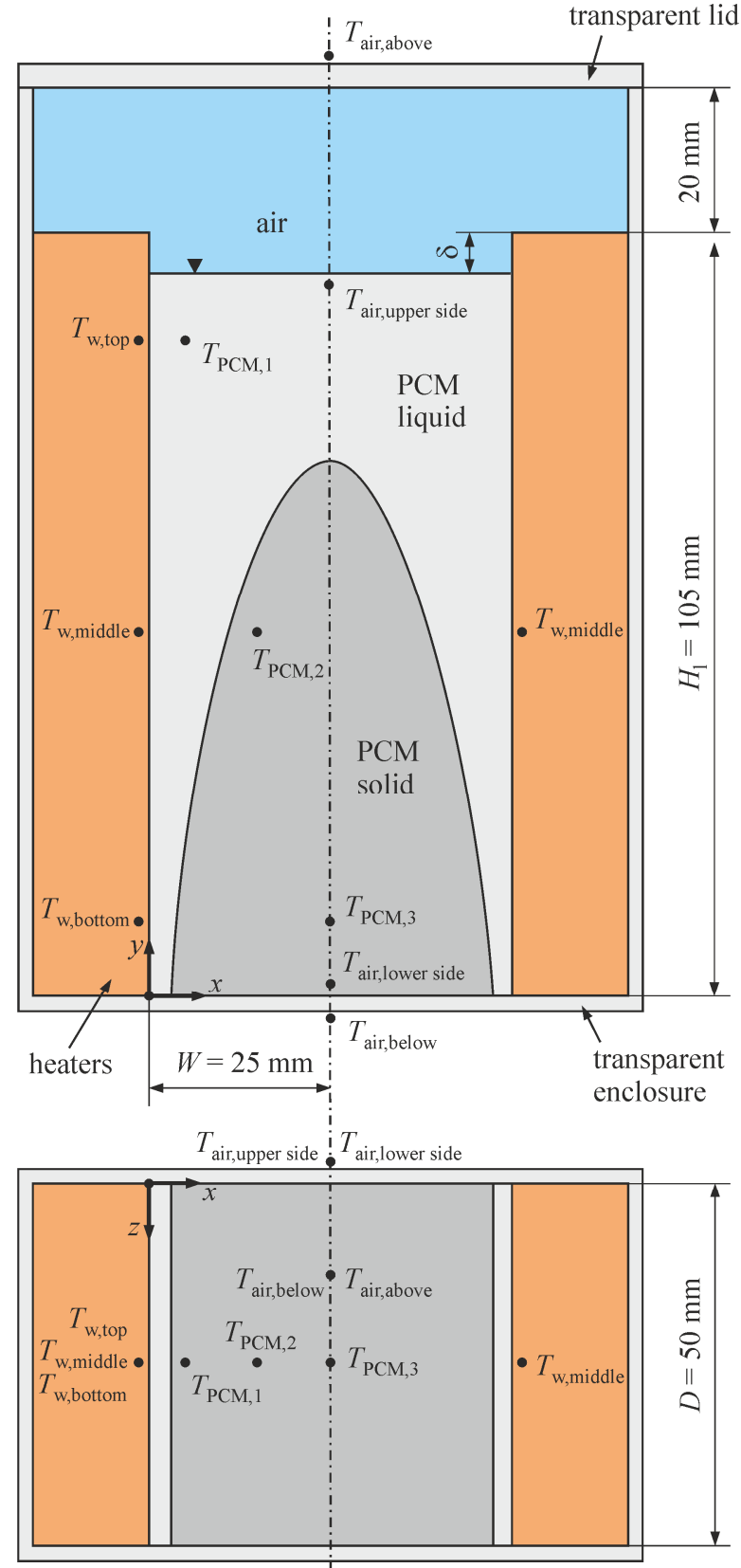

Figure 2: Experimental setup: front view (upper image) and top view (lower image). Relevant thermocouple positions are shown with filled circles.

Temperatures were measured with thermocouples at several positions shown in Figure 2. The three positions given in Table 1 were used for the evaluation of the melting process and the other positons were used to assess boundary conditions.

Table 1: Measurement positions of thermocouples in the PCM used to measure temperatures during the melting process.

\begin{tabular}{lccc}
\hline Thermocouple & PCM,1 & PCM,2 & PCM,3 \\
$\begin{array}{l}\text { Measurement position } \\
(x, y, z) / \mathrm{mm}\end{array}$ & $(5,90,25)$ & $(15,50,25)$ & $(25,10,25)$ \\
\hline
\end{tabular}

The values of the relevant dimensionless numbers, which are the Rayleigh number $R a_{H}$, the Stefan number Ste, the time dependent Fourier number at time of completed melting 
$F_{H}\left(t_{\mathrm{m}}\right)$, the Prandtl number $\operatorname{Pr}$ and the Aspect ratio $A$, are given in Table 2 . With the criterion given by equation (2), the flow was expected to be dominated by natural convection; at least after a certain liquid phase fraction was reached. And, the flow was laminar due to the criterion of equation (3).

Table 2: Values of dimensionless numbers: Rayleigh number $R a$, Stefan number Ste, Fourier number at time of completed melting $\mathrm{Fo}_{H}\left(t_{\mathrm{m}}\right)$, Prandtl number $\mathrm{Pr}$ and aspect ratio $A$.

\begin{tabular}{ccccc}
\hline$R a_{H}$ & Ste & $F o_{H}\left(t_{\mathrm{m}}\right)$ & $P r$ & $A$ \\
\hline $2.0 \cdot 10^{8}$ & 0.092 & 0.11 & 52.2 & 4 \\
\hline
\end{tabular}

\subsection{Material properties}

The material properties of the PCM n-octadecane are given in Table 3. A thorough data set was compiled by Galione et al. [34], from which the properties were adapted.

Table 3: Thermophysical material properties of the PCM n-octadecane adapted from Galione et al. [34].

\begin{tabular}{lccc}
\hline & Unit & $T \leq T_{\mathrm{m}}$ & $T>T_{\mathrm{m}}$ \\
\hline$\rho$ & $\frac{\mathrm{kg}}{\mathrm{m}^{3}}$ & $778.466(\mathrm{~s})$ & $1010.07-$ \\
& $\frac{\mathrm{J}}{\mathrm{kg} \mathrm{K}}$ & $2214.08(\mathrm{l})$ & $1.2463 \cdot 10^{-4} \cdot(T+273.15)^{2}$ \\
$c_{p}$ & $1942(\mathrm{~s})$ & $1394.560+2.7186 \cdot \mathrm{T}$ \\
$k$ & $\frac{\mathrm{W}}{\mathrm{m} \mathrm{K}}$ & $0.15362(\mathrm{~s})$ & $0.2067352-1.841779 \cdot 10^{-4} \cdot \mathrm{T}$ \\
$\mu$ & $\mathrm{Pa} \mathrm{s}$ & - & 0.02966723 \\
$\beta$ & $\frac{1}{\mathrm{~K}}$ & - & $-8.533286 \cdot 10^{-5} \cdot(T+273.15)$ \\
$T_{\mathrm{m}}$ & ${ }^{\circ} \mathrm{C}$ & & $8.9 \cdot 10^{-4}$ \\
& $\frac{\mathrm{kJ}}{\mathrm{kg}}$ & & 242.454 \\
\hline
\end{tabular}

The properties depending on temperature $T$ and liquid phase fraction $f_{\mathrm{l}}$ are the density $\rho$, the specific isobaric heat capacity $c_{p}$ and the thermal conductivity $k$. Constant values are given for the solid state $T<T_{\mathrm{m}}$, which was sufficiently accurate due to the low subcooling of $1 \mathrm{~K}$ in this test case. The value in the liquid state at melting temperature $T=T_{\mathrm{m}}$ is also given. Temperature-dependent values are given for the liquid phase with $T>T_{\mathrm{m}}$. The dynamic viscosity $\mu$ is only specified in the liquid phase and the expression by Galione et al. [34] was linearized for the temperature region of this investigation. The melting temperature $T_{\mathrm{m}}$ and the latent heat $L$ are also given by Galione et al. [34]. The thermal expansion coefficient was derived from a mean density $\bar{\rho}$ between the solid and liquid state and the density $\rho_{\mathrm{l}}$ in the liquid region with $T>T_{\mathrm{m}}$ :

$\beta=-\left.\frac{1}{\bar{\rho}} \frac{\partial \rho_{\mathrm{l}}}{\partial T}\right|_{T_{\mathrm{m}}}$.

The thermophysical properties of the steel heaters used to heat up the PCM, the PLEXIGLAS ${ }^{\circledR}$ enclosure, in which the
PCM is filled, and the properties of ambient air are given in Table 4.

Table 4: Thermophysical properties of enclosure materials and ambient air.

\begin{tabular}{ccccc}
\hline Property & Unit & $\begin{array}{c}\text { Steel heaters } \\
1.4301\end{array}$ & $\begin{array}{c}\text { PLEXIGLAS }^{(\beta)} \\
\text { enclosure }\end{array}$ & Air \\
\hline$\rho$ & $\frac{\mathrm{kg}}{\mathrm{m}^{3}}$ & 7900 & 1180 & 1.225 \\
$c_{p}$ & $\frac{\mathrm{J}}{\mathrm{kg} \mathrm{K}}$ & 500 & 1470 & 1006 \\
$k$ & $\frac{\mathrm{W}}{\mathrm{m} \mathrm{K}}$ & 15 & 0.19 & 0.0242 \\
$\mu$ & Pa s & - & - & $1.79 \cdot 10^{-5}$ \\
\hline
\end{tabular}

\section{Numerical model}

We developed two different numerical models with varying degree of detail and different simplifications. Our models were based on the commercial software package ANSYS ${ }^{\circledR}$ Fluent 16, where the Navier-Stokes equations including the energy equation and a source term enthalpy method were solved on a fixed finite volume grid. A first detailed model used variable material properties and the volume of fluid (VOF) method to model volume expansion of the PCM into an additional air phase, and it was solved in two dimensions (V-2D). A second simplified model used the Boussinesq approximation and constant material properties and thereby neglected volume expansion. It was solved in either two dimensions (B-2D) or three dimensions (B-3D).

\subsection{Model simplifications}

The following simplifications were used by both models: 1) the flow in the liquid phase of the PCM was incompressible and Newtonian, 2) the sharp interface between the solid and liquid phase of the pure PCM or eutectic mixture of multiple PCMs could be represented by a narrow so called mushy region, where the material was neither solid nor liquid but a mixture of both phases, 3) natural convection in the liquid phase of the PCM was laminar and 4) radiation and viscous dissipation in the liquid phase of the PCM were negligible.

There are further simplifications that were different for the two models. The detailed model (V-2D) introduced an interface between the PCM and an air phase to allow for volume expansion. However, it is assumed that 6) the effect of surface tension on the interface between liquid PCM and air was negligible. The simplified models (B-2D and B-3D) used a constant density and the Boussinesq approximation and therefore assumed that 7) the Boussinesq approximation was valid in this case and density change (or volume expansion) of the PCM during melting was negligible. Furthermore, these models assumed that 8) constant thermophysical properties of the PCM were sufficiently accurate. 


\subsection{Modeling of material properties}

The different modeling of material properties in the detailed model with VOF method (V-2D) and in the Boussinesq models (B-2D and B-3D) is compared in Table 5.

Table 5: Modeling of material properties in the detailed model (V-2D) and in the simplified models (B-2D and B-3D).

\begin{tabular}{lcc}
\hline Property & V-2D & B-2D, B-3D \\
\hline Density $\rho$ & $\rho\left(f_{\mathrm{l}}, T\right)$ & $\bar{\rho}$ \\
Specific heat capacity $c_{p}$ & $c_{p}(T)$ & $\bar{c}_{p}$ \\
Thermal conductivity $k$ & $k\left(f_{\mathrm{l}}, T\right)$ & $k_{\mathrm{l}}$ \\
Dynamic viscosity $\mu$ & $\mu(T)$ & $\mu\left(T_{\mathrm{m}}+\frac{\Delta T}{2}\right)$ \\
\hline
\end{tabular}

In the detailed model (V-2D), the density was constant in the solid state and during phase change it linearly varied with the liquid phase fraction between the solid and liquid state:

$$
\rho\left(f_{\mathrm{l}}, T\right)=f_{\mathrm{l}} \rho_{\mathrm{l}}+\left(1-f_{\mathrm{l}}\right) \rho_{\mathrm{s}} .
$$

The specific heat capacity could not be specified depending on the liquid phase fraction in ANSYS $^{\circledR}$ Fluent; it had to depend on temperature. Even with the appropriate user defined function (UDF) macro this was not possible. So, we introduced a small temperature range in the phase change region between $T_{\mathrm{s}}=T_{\mathrm{m}}-0.1 \mathrm{~K}$ and $T_{\mathrm{l}}=T_{\mathrm{m}}+0.1 \mathrm{~K}$ in which the specific heat capacity changed linearly:

$$
c_{p}(T)=c_{p, \mathrm{~s}}+\frac{c_{p, 1}-c_{p, \mathrm{~s}}}{T_{1}-T_{\mathrm{s}}}\left(T-T_{\mathrm{s}}\right)
$$

The thermal conductivity was constant in the solid state and during phase change it depended on the liquid phase fraction

$$
k\left(f_{\mathrm{l}}, T\right)=f_{\mathrm{l}} k_{\mathrm{l}}+\left(1-f_{\mathrm{l}}\right) k_{\mathrm{s}} .
$$

In the liquid state, the properties $\rho, c_{p}, k$ depended on temperature. The dynamic viscosity $\mu$ was only defined in the liquid state and also depended on temperature.

With the Boussinesq approximation in the simplified models (B-2D and B-3D), the density was assumed constant in all terms but the linearized Buoyancy term in the momentum equation. We chose the mean value of the liquid and the solid state near the melting point as constant mean density $\bar{\rho}$. From a fluid dynamics viewpoint, the density in the liquid state would have been the optimal choice, because the melting process is dominated by natural convection in the liquid phase. However, the amount of sensible and latent energy stored in the initial solid state would have been significantly lower than in reality. With a mean density $\bar{\rho}$ and a mean PCM volume, the amount of stored energy was correct in a mean sense. For the same reason, we also chose a mean specific heat capacity $\bar{c}_{p}$. For the thermal conductivity $k$, we chose the value in the liquid state, because temperature gradients and heat conduction mostly occurred in the liquid phase while the solid phase was nearly isothermal throughout the melting process. The dynamic viscosity was set to a constant value evaluated at the mean liquid temperature $T_{\mathrm{m}}+\frac{\Delta T}{2}$.

The material properties density $\rho$, specific heat capacity $c_{p}$, thermal conductivity $k$ and dynamic viscosity $\mu$ of $n-$ octadecane are plotted over temperature in Figure 3. The variable properties used by the detailed model (V-2D) are shown with solid lines and the constant properties used by the simplified models (B-2D, B-3D) are represented with dashed lines.
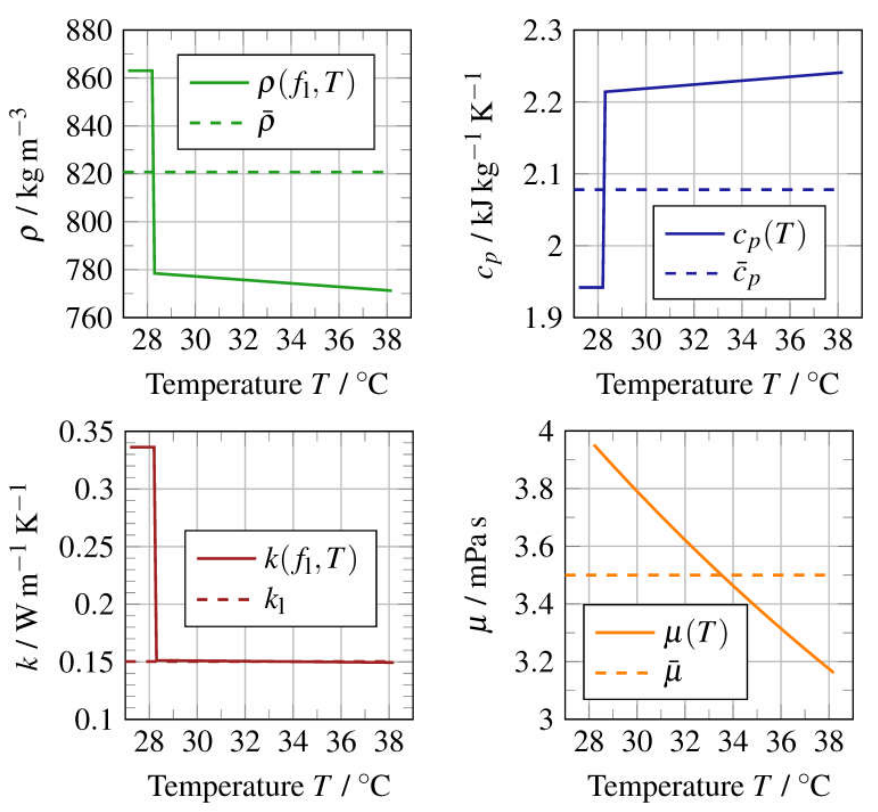

Figure 3: The density $\rho$, specific heat capacity $c$, thermal conductivity $k$ and dynamic viscosity $\mu$ of n-octadecane plotted over temperature with solid lines for the detailed model (V-2D) and dashed lines for the simplified models (B-2D, B-3D).

\subsection{Governing equations}

For the two different models, different sets of governing equations were needed. The detailed model (V-2D) maintained a variable density and used the VOF method while the simplified models (B-2D, B-3D) assumed a constant density and included buoyancy with the Boussinesq approximation, which introduces a linearized temperature-dependent buoyancy term in the momentum equations.

\subsubsection{Detailed VOF model with air phase (V-2D)}

The conservation equations of mass, momentum and energy are solved with one set of equations for the two phases of PCM and air with the volume of fluid method [35]: the continuity equation is solved only for the secondary phase, which is the PCM,

$$
\begin{aligned}
\frac{1}{\rho}\left[\frac{\partial}{\partial t}\left(\alpha_{\mathrm{PCM}} \rho\right)+\right. & \nabla \cdot\left(\alpha_{\mathrm{PCM}} \rho \mathbf{u}\right) \\
& \left.=\dot{m}_{\mathrm{air} \rightarrow \mathrm{PCM}}-\dot{m}_{\mathrm{PCM} \rightarrow \text { air }}\right],
\end{aligned}
$$

where $\alpha_{\mathrm{PCM}}$ is the volume fraction of the PCM, $\rho$ is the density and $\mathbf{u}=(u, v, w)^{\top}$ is the flow velocity in $x, y$ and $z$-direction, respectively. The volume fraction of the primary phase, which is air, is determined with the following constraint:

$\alpha_{\mathrm{PCM}}+\alpha_{\text {air }}=1$.

The momentum equation with buoyancy term $\mathbf{F}_{\mathrm{b}}$ and a momentum source term $\mathbf{S}_{\mathbf{u}}$ is

$\frac{\partial}{\partial t}(\rho \mathbf{u})+\nabla \cdot\left(\rho \mathbf{u} \mathbf{u}^{\top}\right)=\nabla \cdot \mathbf{\tau}-\nabla p+\mathbf{F}_{\mathbf{b}}+\mathbf{S}_{\mathbf{u}}$ 
where $p$ is the pressure. The stress tensor $\boldsymbol{\tau}$ is given as

$\mathbf{\tau}=\mu\left[\left(\nabla \mathbf{u}+\nabla \mathbf{u}^{\top}\right)-\frac{2}{3} \nabla \cdot \mathbf{u I}\right]$,

where $\mathbf{I}$ is the unit tensor. The buoyancy term is

$\mathbf{F}_{\mathrm{b}}=\rho g \hat{\mathbf{e}}_{y}$,

where $g$ is the gravity constant and $\hat{\mathbf{e}}_{y}$ is a unit vector in $y$ direction.

The energy equation for the specific enthalpy $h$ with an enthalpy source term $S_{h}$ is

$\frac{\partial}{\partial t}(\rho h)+\nabla \cdot(\rho \mathbf{u} h)-\nabla \cdot(k \nabla T)=S_{h}$.

\subsubsection{Simplified Boussinesq models ( $B-2 D, B-3 D)$}

The conservation equations of mass, momentum and energy are solved without volume expansion with the Boussinesq approximation [35]: The continuity equation with constant density is

$\nabla \cdot \mathbf{u}=0$.

The momentum equation with buoyancy term $\mathbf{F}_{\mathrm{b}}$ and a momentum source term $\mathbf{S}_{\mathbf{u}}$ is

$\bar{\rho} \frac{\partial \mathbf{u}}{\partial t}+\bar{\rho}(\mathbf{u} \cdot \nabla) \mathbf{u}=\mu \nabla^{2} \mathbf{u}-\nabla p+\mathbf{F}_{\mathrm{b}}+\mathbf{S}_{\mathbf{u}}$

where $\bar{\rho}$ is the constant mean density defined in section 5.2. With the Boussinesq approximation, the buoyancy term is

$\mathbf{F}_{\mathrm{b}}=g \bar{\rho} \beta\left(T-T_{\mathrm{m}}\right) \hat{\mathbf{e}}_{y}$.

The buoyancy depends on the thermal expansion coefficient $\beta$ and a temperature difference to a reference temperature, at which the constant density was specified. In this case this is the melting temperature $T_{\mathrm{m}}$.

The energy equation for the specific enthalpy $h$ with an enthalpy source term $S_{h}$ is

$\bar{\rho} \frac{\partial h}{\partial t}+\bar{\rho} \nabla \cdot(\mathbf{u} h)-\nabla \cdot(k \nabla T)=S_{h}$.

\subsubsection{Enthalpy-Porosity model}

The energy equation (13) or (17) is transformed with the enthalpy-porosity method [13-15]. In the following, we will use the more general equation (13). The central idea of the method is to write the enthalpy $h$ as the sum of the sensible enthalpy $h_{\text {sens }}$ and the latent enthalpy $h_{\text {lat }}$ :

$h=h_{\text {sens }}+h_{\text {lat }}$.

The sensible enthalpy is

$h_{\text {sens }}(T)=\int_{T_{\text {ref }}}^{T} c_{p} d T^{\prime}$

and the latent enthalpy is the product of the latent heat of fusion $L$ and the liquid phase fraction $f_{1}$ :

$h_{\text {lat }}=L f_{1}$.
The liquid phase fraction is in general defined by the temperature $T$ in relation to the solidus temperature $T_{\mathrm{s}}$ and the liquidus temperature $T_{1}$ :

$f_{\mathrm{l}}=\left\{\begin{array}{cc}0, & T \leq T_{\mathrm{s}} \\ 0 \ldots 1, & T_{\mathrm{s}}<T<T_{1}, \\ 1, & T \geq T_{\mathrm{l}}\end{array}\right.$

In the so called mushy region, $0<f_{1}<1$, the material is neither solid nor liquid, but in a state of melting or solidification. In this region, a relationship between liquid phase fraction and temperature has to be defined.

ANSYS $^{\circledR}$ Fluent offers two variations of the enthalpyporosity technique: the melting point model [13] and the melting range model [15]. The melting point model is activated in the software, when the solidus and liquidus temperature are specified as the same value, which is the melting temperature $T_{\mathrm{m}}$, and the melting range model is applied when a higher liquidus than solidus temperature is specified [35]. The melting range model could also be applied for a material with a melting point, when a small melting range is selected. The advantage in modeling with a melting range would be that all material properties can depend on temperature. Instead, with the melting point model, the properties have to depend on the liquid phase fraction, which is implemented with user defined functions (UDFs), because the temperature does not change during phase change. Both models were tested and the melting point model was preferred, because it showed more plausible results and better agreement with experiments.

In the melting point model [13], a linear relationship over a small temperature range of $2 \xi$ between $T_{\mathrm{s}}=T_{\mathrm{m}}-\xi$ and $T_{1}=T_{\mathrm{m}}+\xi$ is introduced and the liquid phase fraction is then defined as:

$f_{\mathrm{l}}=\left\{\begin{array}{cc}0, & T \leq T_{\mathrm{m}}-\xi \\ \frac{T-\left(T_{\mathrm{m}}-\xi\right)}{2 \xi}, & T_{\mathrm{m}}-\xi<T<T_{\mathrm{m}}+\xi, \\ 1, & T \geq T_{\mathrm{m}}+\xi\end{array}\right.$

After introducing equation(18) in (13), dropping the subscript sens, and defining the energy equation source term as

$S_{h}=L\left(\frac{\partial}{\partial t}\left(\rho f_{1}\right)+\nabla \cdot\left(\rho \mathbf{u} f_{\mathrm{l}}\right)\right)$,

the original form of the energy equation (13) is obtained with the latent enthalpy being expressed in the source term:

$$
\begin{aligned}
\frac{\partial}{\partial t}(\rho h)+\nabla \cdot(\rho \mathbf{u} h) & -\nabla \cdot(k \nabla T) \\
= & L\left(\frac{\partial}{\partial t}\left(\rho f_{1}\right)+\nabla \cdot\left(\rho \mathbf{u} f_{1}\right)\right)
\end{aligned}
$$

To modify the velocities in the mushy region and in the solid [13], another source term is introduced into the momentum equations (10) or (15),

$\mathbf{S}_{\mathbf{u}}=-B\left(f_{\mathrm{l}}\right) \mathbf{u}$,

where a parameter $B\left(f_{1}\right)$, which depends on the liquid phase fraction, is multiplied with the velocity vector. This parameter has to be zero in the liquid phase to allow for free motion. When it takes large values in the solid phase, the velocities are 
forced to near zero values in the linear system of equations of an implicit method [14]. While different functions fulfil this requirement, most often the Carman-Kozeny equation, which is derived from the Darcy law for fluid flow in porous media [36], is used in a modified form [35]:

$B\left(f_{\mathrm{l}}\right)=C \frac{\left(1-f_{\mathrm{l}}\right)^{2}}{f_{\mathrm{l}}{ }^{3}+q}$.

The original Carman-Kozeny equation would yield infinity if the liquid phase fraction approached zero. To limit $B\left(f_{\mathrm{l}}\right)$ to numerically applicable finite values, a constant value $q$ is additionally added in the denominator. In ANSYS ${ }^{\circledR}$ Fluent, the value is fixed to $q=10^{-3}$. The parameter $C$ is called the mushy region or mushy zone constant and is a model constant, which replaces the physical properties in the Carman-Kozeny equation. It has to be adjusted to the problem, because it will influence the morphology of the mushy region [13]. Investigations on the influence of the value $C$ were described by Shmueli et al. [22]. In the present study, a value of $10^{6}$ was used. We found that, using a melting point material, the solution is rather insensitive to this parameter compared to a melting range material. With a melting point material, the mushy zone is expected to be narrow, approximately as wide as a computational cell, and to exist only due to the discretization error.

\subsection{D domains and initial and boundary conditions}

The domain contained either a zone for the mixture of PCM and air in the case of the detailed model with VOF method (V2D) or a zone of only PCM in the case of the simplified model with Boussinesq approximation (B-2D). Additionally, a small part of heater material with $1 \mathrm{~mm}$ thickness and the bottom part of the acrylic glass enclosure with $2 \mathrm{~mm}$ wall thickness are included in the domain. The domain only extended to half of the enclosure and the symmetry to the mid-plane was used. After comparing a simulation with full enclosure width to another simulation with half width and symmetry condition, the flow and melting was found to be indistinguishable. Although the two models V-2D and B-2D were created as similar as possible, there were differences in the domain and the initial and boundary conditions. Both 2D models are shown in Figure 4.

The domain of the detailed model (V-2D) was slightly larger to accommodate an additional air phase on top of the PCM that allowed volume expansion of the PCM during melting. The air was assumed incompressible with constant properties. A pressure outlet allowed the air to flow out of the domain. This boundary condition additionally needed the specification of a backflow temperature $T_{\mathrm{bf}}=T_{\mathrm{w}}$ and a backflow volume fraction for the PCM phase $\alpha_{\mathrm{PCM}, \mathrm{bf}}=0$. With setting the backflow temperature higher than the PCM temperature, heat was not transferred out of the domain by convection; only a negligible conduction heat flow into the domain might have occurred. Setting the backflow volume fraction correctly is important, so that only air and not PCM can flow into the system. The initial conditions had to be patched in ANSYS $^{\circledR}$ Fluent, i.e. firstly the domain was initialized with setting $u_{0}, v_{0}, p_{0}, T_{0}$ and $\alpha_{\text {air }, 0}=0$. Then only the air region was patched with setting $\alpha_{\text {air }, 0}=1$.

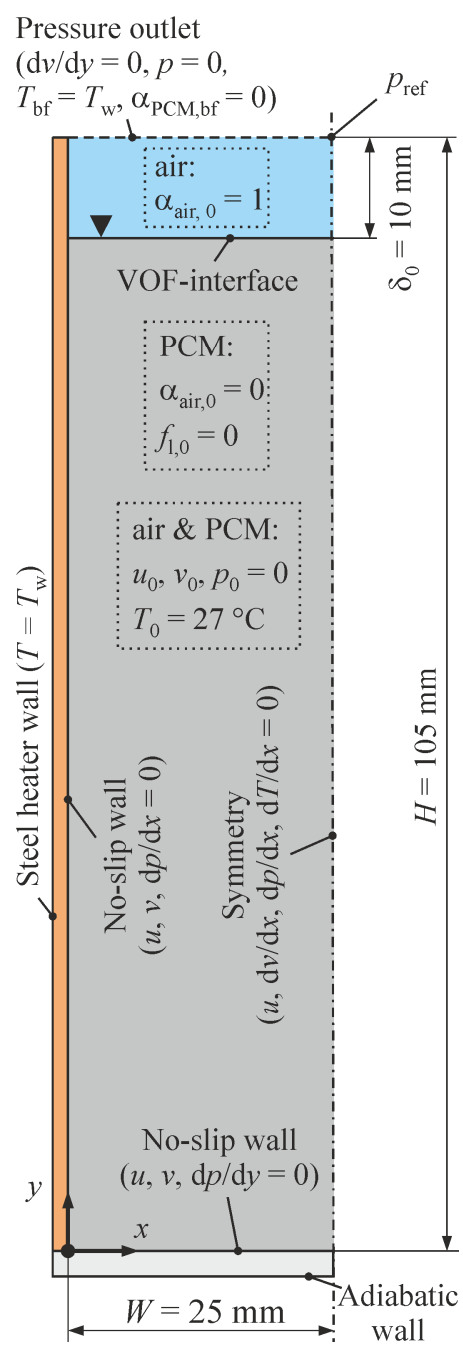

a) VOF model (V-2D)
Symmetry

$(v, \mathrm{~d} v / \mathrm{d} y, \mathrm{~d} p / \mathrm{d} y, \mathrm{~d} T / \mathrm{dy}=0)$

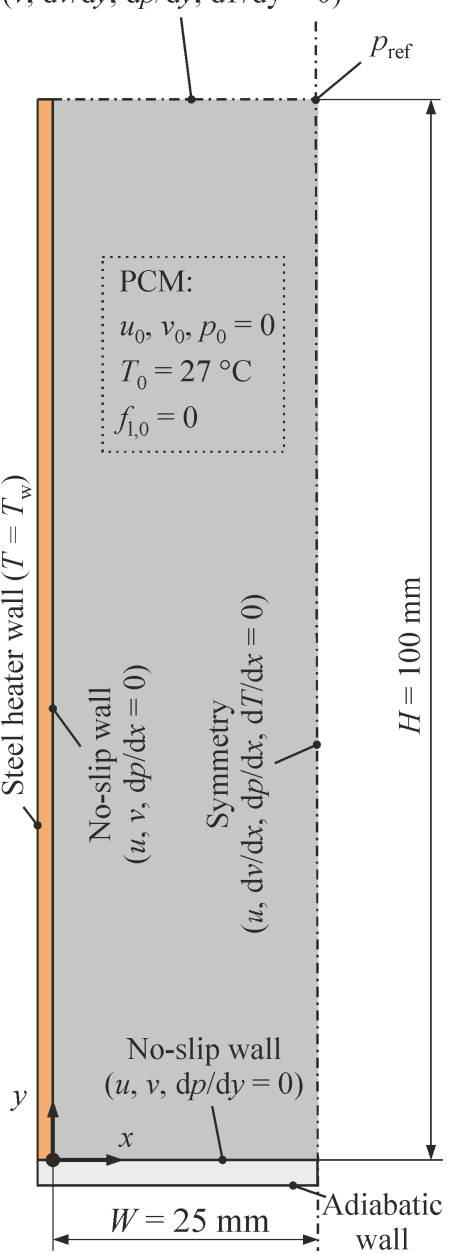

b) Boussinesq model (B-2D)
Figure 4: Domain, initial and boundary conditions of the detailed model with VOF method (V-2D) and the simplified model with Boussinesq approximation (B-2D).

The simplified model (B-2D) did not include an air phase for volume expansion. The height of the domain was set to the average between the liquid and the solid state and therefore was smaller than in the V-2D model. Instead, the top side was modeled with a symmetry boundary condition, i.e. all gradients and the velocity component in $y$-direction were zero. The domain was initialized with setting $u_{0}, v_{0}, p_{0}$ and $T_{0}$.

For both these 2D models, all outer sides except the steel heater wall and the pressure outlet were adiabatic and thus heat exchange with the environment was neglected.

\subsection{D Boussinesq model with boundary effects (B-3D)}

The simplified model (B-2D) was extended to a 3D model with boundary effects (B-3D). The 3D domain had the same dimensions in the $x-y$-plane as the 2D domain. A second symmetry plane was used in $z$-direction and thus the depth was the half depth of the symmetric PCM region with $D / 2=$ $25 \mathrm{~mm}$. An additional acrylic glass window with a thickness of $2 \mathrm{~mm}$ enclosed the PCM at the back side. The domain of the B$3 \mathrm{D}$ model is illustrated in Figure 5. 


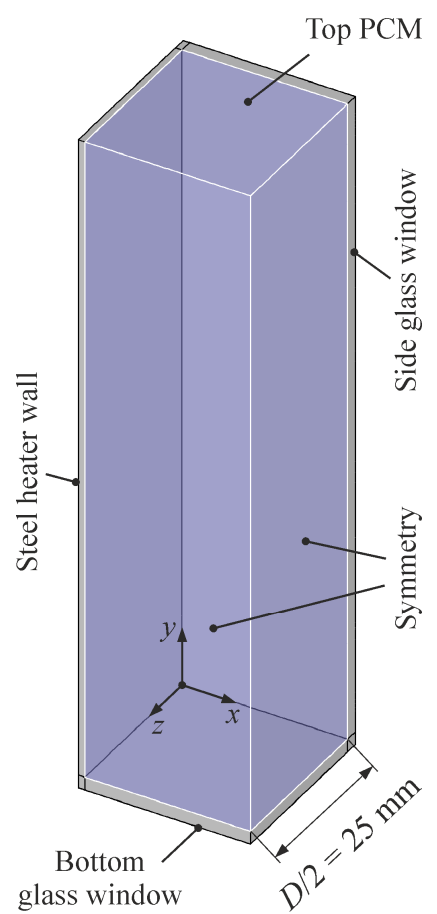

Figure 5: Boussinesq model in 3D (B-3D) including viscous boundary layer effects and thermal boundary conditions.

In this $3 \mathrm{D}$ model, other than in the $2 \mathrm{D}$ models, viscous boundary layer effects of the front and back walls were included. Additionally, thermal boundary conditions were imposed with measured data from the validation experiment by Vogel and Bauer [3]. While the simulation cannot model all physical effects of an experiment, the experiment is not able to perfectly reproduce the ideally stated physical problem with isothermal walls and adiabatic boundaries stated in section 3 . With imposing the inevitable boundary effects of the experiment onto the simulation, a comparison of simulation and experiment free of the influence of boundary effects was possible. The thermal boundary conditions are summarized in Table 6 . The positions of thermocouples are found in Figure 2.

Table 6: Thermal boundary conditions in the 3D model.

\begin{tabular}{lc}
\hline Boundary & Thermal boundary condition \\
\hline $\begin{array}{l}\text { Steel heater } \\
\text { wall }\end{array}$ & $T_{\mathrm{w}}(t, y)=f_{\mathrm{i}}\left(T_{\mathrm{w}, \text { top }}(t), T_{\mathrm{w}, \text { bottom }}(t)\right)$ \\
Top PCM & $\dot{Q}_{\text {top }}(t)=\dot{Q}_{\text {conv }+ \text { rad }}\left(T_{\text {air,above }}(t)\right)$ \\
Bottom glass & $T_{\text {bottom }}(t)=T_{\text {air,below }}(t)$ \\
window & $T_{\text {side }}(t, y)=f_{\mathrm{i}}\left(T_{\text {air,lower side }}(t), T_{\text {air,upper side }}(t)\right)$ \\
window & wilass
\end{tabular}

Firstly, two experimentally measured temperatures at the heater wall $T_{\mathrm{w} \text {,top }}$ and $T_{\mathrm{w} \text {,bottom }}$ were interpolated over time $t$ and over height $y$ onto the simulation time and boundary mesh, and then used as temperature boundary condition at the steel heater wall. Secondly, a heat flow through the top of the PCM was modeled with a mixed boundary condition in ANSYS ${ }^{\circledR}$ Fluent. With this boundary condition, a heat flow by heat radiation and by natural convection in the adjacent air gap over the PCM was modeled. In the experiment, this air gap was enclosed on top with another acrylic glass plate on which the temperature $T_{\text {air,above }}$ was measured. This temperature was interpolated to the simulation time from the experimental data and set as free stream boundary temperature and external radiation temperature. The external emissivity for the radiation part was set to a value of 0.9 . The heat transfer coefficient for the convective part was calculated with an approximate effective thermal conductivity model for natural convection in an air enclosure [37]. Thirdly, the heat flow by heat conduction through the side and bottom glass windows was included with temperature boundary conditions. In the experiment, a temperature at the outer side of the bottom window $T_{\text {air,below }}$ was measured, which was interpolated in time and set as boundary temperature in the simulation. At the side glass window, temperatures were measured at two positions at the bottom and top of the outer side of the window, $T_{\text {air,lower side }}$ and $T_{\text {air,upper side. These temperatures were first interpolated in }}$ time and then over the height $y$ onto the boundary mesh of the simulation. These transient boundary conditions were calculated in MATLAB, which was coupled to ANSYS Fluent, as already described by Vogel et al. [38]. The presented 3D model was an accurate reproduction of the experiment and its boundary conditions. It was mainly used to validate the numerical model with the experiment.

\subsection{Discretization}

The governing equations were discretized with a pressurebased finite volume method [39] and implicit time integration with ANSYS ${ }^{\circledR}$ Fluent 16 [40]. The segregated solver was used with the PISO method for pressure-velocity-coupling [39]. The second order derivatives in the diffusive terms were approximated by second order central differences, the first order derivatives in the convective terms with a second order upwind scheme [40]. The interpolation of pressure values at the cell faces was done with the PRESTO! scheme [39]. For the VOF method in the detailed model (V-2D), the explicit scheme was used with a Courant number of 0.25 . The implicit body force formulation and the sharp interface modeling were selected. The volume fraction was calculated with the geometric reconstruction scheme [40]. The resulting linear systems were solved with an iterative method with algebraic multigrid acceleration [39].

The rectangular geometries were discretized with ANSYS ${ }^{\circledR}$ meshing [40] on a structured quad mesh in 2D or a structured hex mesh in 3D. A cell size of $0.25 \mathrm{~mm}$ was found to be optimal in a mesh dependency study. Therefore, this size was used throughout the 2D meshes, which resulted in meshes with about 40000 control volumes. However, for the 3D case, the cell sizes were increased to decrease the computational effort. In the $x$-direction, the cell size remained $0.25 \mathrm{~mm}$ to resolve the boundary layers. In the $y$ - and $z$-direction the cell size was increased to a range of $0.25 \ldots 1 \mathrm{~mm}$ and a cell size distribution with a finer mesh towards the boundaries was introduced. The 3D mesh was then built of about 140000 control volumes.

For the simplified models (B-2D, B-3D), a time step of $\Delta t=0.1 \mathrm{~s}$ was a good tradeoff between fast convergence and low computation time. The maximum Courant Number was slightly above one. The implicit scheme theoretically allowed larger Courant numbers, but we found that convergence became inefficiently slow. A smaller time step was needed for convergence of the detailed model with VOF method (V-2D). 
We found the best results with the variable time stepping method and a time step corresponding to a Courant Number of 0.25 , which was the same as specified for the explicit scheme in the VOF model. With this method the time step was of the order of $10^{-4} \mathrm{~s}$ at the beginning and it increased up to a specified maximum of $10^{-2} \mathrm{~s}$ at later times.

Iterations continued until a convergence criterion was met. Solution convergence was found to be restricted by the mass continuity, for which the scaled absolute residual threshold was set to a value of $10^{-3}$. Between 30 and 150 iterations were needed to reach this criterion. The residuals of the momentum equations and the energy equation fell below $10^{-8}$ and $10^{-15}$, respectively.

\section{Results and discussion}

In the last section, we presented a 2D Boussinesq model (B2D) and a 2D VOF model (V-2D), both with ideal boundary conditions, and a 3D Boussinesq model (B-3D) adjusted to experimentally measured boundary conditions. In this section, we first compare the 2D models (V-2D, B-2D) with each other to find out to what extent the Boussinesq approximation and constant material properties are valid simplifications. Then, we compare the 3D Boussinesq model including boundary effects (B-3D) to the benchmark experiment.

\subsection{Comparison of the $2 D$ models $V-2 D$ and $B-2 D$}

To compare the two models in $2 \mathrm{D}$, we analyzed the melting process qualitatively with the $2 \mathrm{D}$ fields of phase state and velocity. And, we quantitatively evaluated the liquid phase fraction and heat flow rate over time, the velocity extrema occurring in the liquid phase over time, and the temperatures at three positions over time.

\subsubsection{D fields of phase state and velocity}

The fields of phase state and velocity by natural convection in the liquid phase are illustrated in Figure 6 at times $t=$ $3600 \mathrm{~s}$ and $t=7200 \mathrm{~s}$. The solid phase of the PCM is drawn in black such as the solid side and bottom walls. In the liquid phase, filled contours show the velocity magnitude and vectors show the velocity direction as well as the magnitude. On the left side of each plot, the VOF-model (V-2D) is shown and on the right side, the Boussinesq model (B-2D) is shown. The interface between the PCM and the air phase in the V-2D model is represented by a white line, while, in the B-2D model, the top side is a fixed slip wall.

The velocity magnitudes and the boundary layers are qualitatively similar in both models. However, the resulting solid phase shape appears to be smaller and wider in the V-2D model compared to the taller and narrower solid phase in the B-2D model. This indicates a faster melting at the top and slower melting at the bottom in the V-2D model relative to the B-2D model. This difference may be either due to the different choice in material properties or due to the different boundary condition at the top, namely the VOF-interface or the fixed slip wall. A pre-study already showed that the influence of variable heat capacity, thermal conductivity and viscosity is rather small. The volume expansion with density change and the moving interface in the VOF model may be the main reason for the observed differences. An obvious reason is that the solid phase had an initial height of only $H_{\mathrm{s}}=95 \mathrm{~mm}$ in the $\mathrm{V}-2 \mathrm{D}$ model instead of $H=100 \mathrm{~mm}$ in the B-2D model, which made a difference in height from the beginning. The volume expansion up to a final height of $H_{1}=105 \mathrm{~mm}$ in the V-2D model could have also increased convection at the top and inhibited convection at the bottom, due to an additional overall upward flow that increased the upward flow at the heated walls and decreased the downward flow at the solid phase. The result is probably a stronger convection at the top than at the bottom, which leads to the difference in the phase front shapes. 


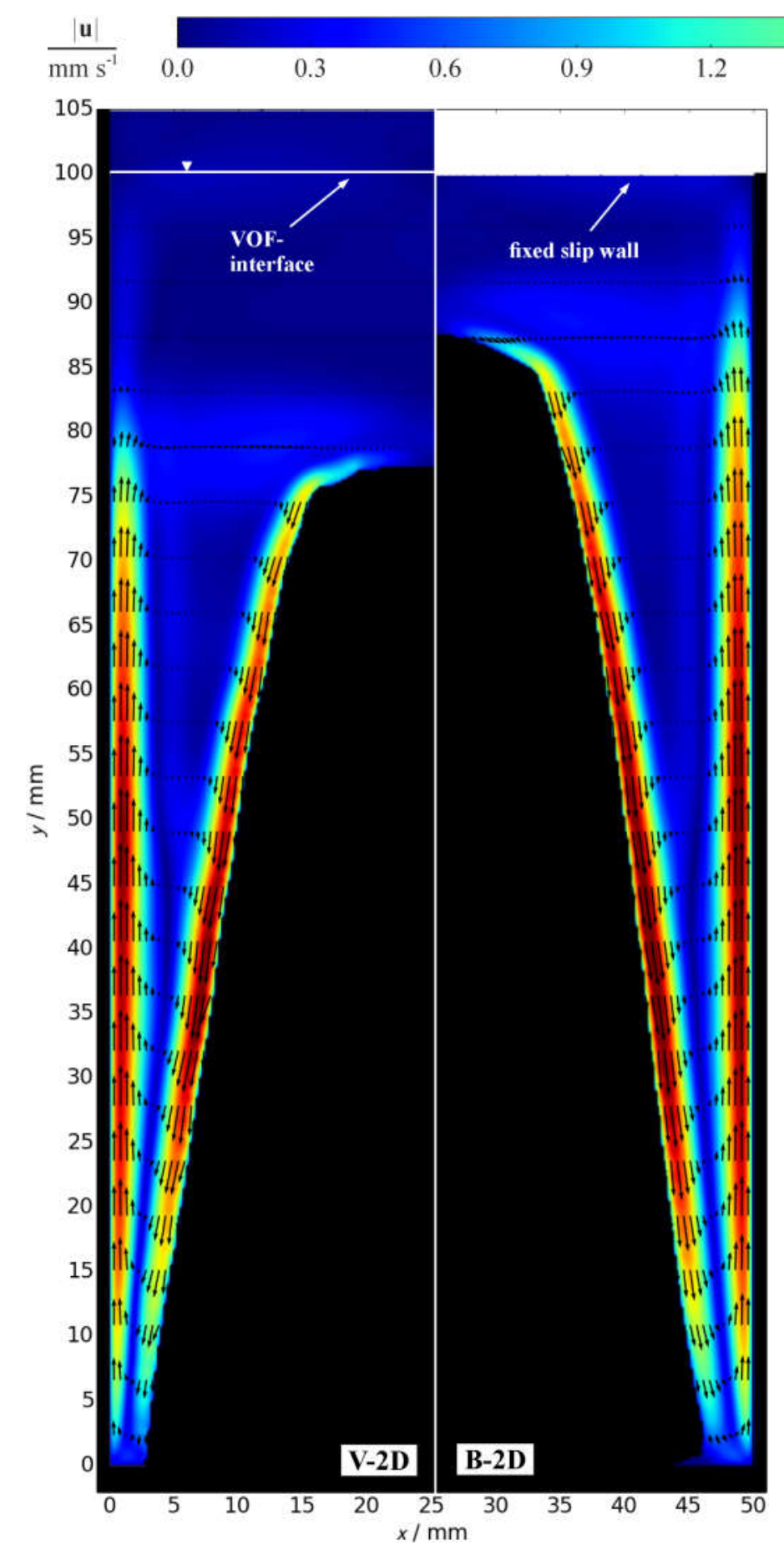

a) $t=3600 \mathrm{~s}$

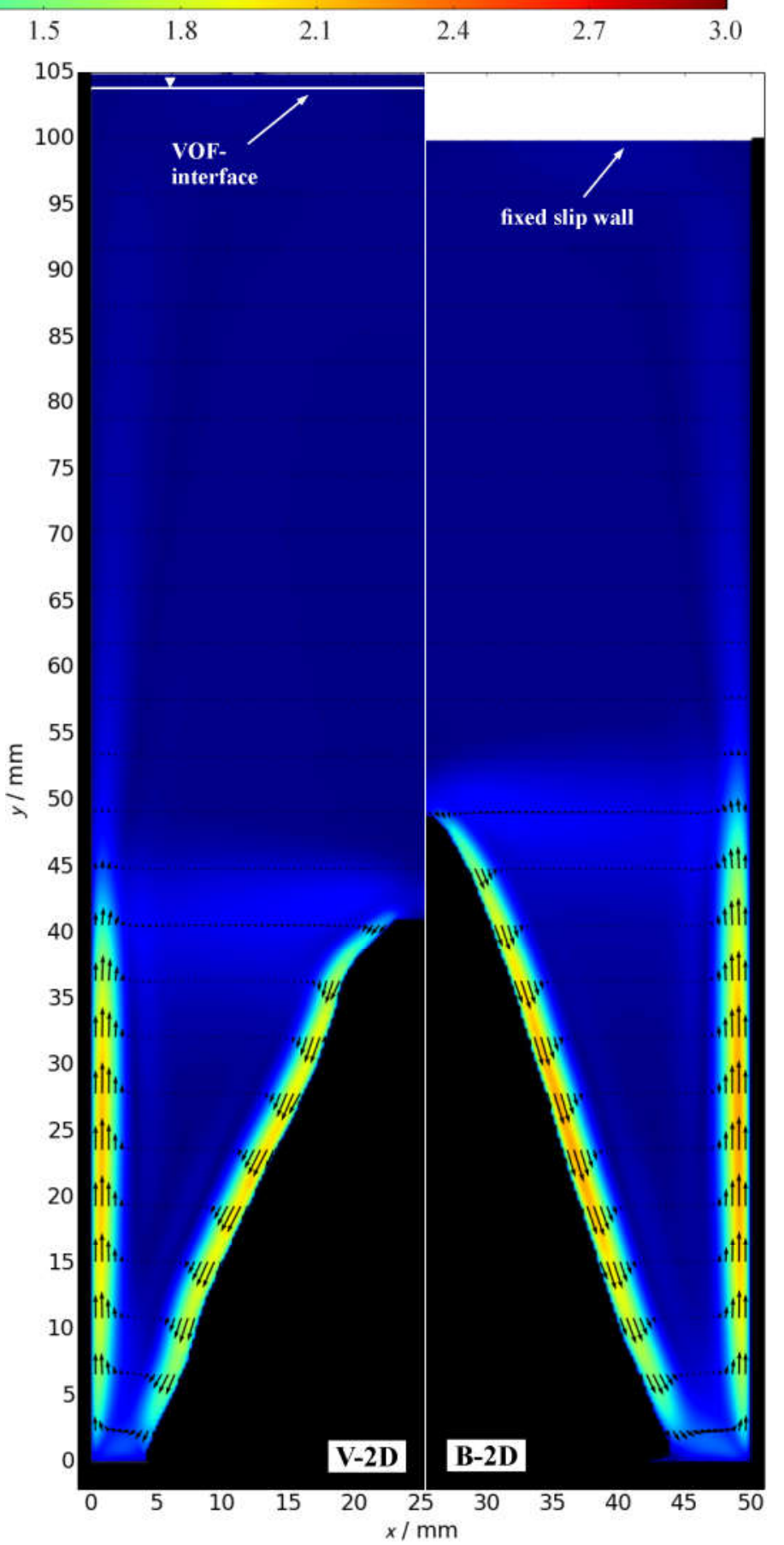

b) $t=7200 \mathrm{~s}$

Figure 6: Comparison of the 2D fields of phase state and velocity for the 2D Boussinesq model (B-2D) and for the 2D VOF model (V-2D) at two different time steps $\left(R a_{H}=2.0 \cdot 10^{8}\right.$, Ste $\left.=0.092, P r=52.2, A=4\right)$. The solid phase of the PCM is drawn in black and velocities in the liquid phase are illustrated by contours of velocity magnitude and vectors scaled by velocity magnitude. 


\subsubsection{Liquid phase fraction and heat flow rate}

A quantitative analysis of the liquid phase fraction and the heat flow rate helps to further understand the differences in the melting process. Figure 7 shows both quantities plotted over time for the VOF model (V-2D) and for the Boussinesq model (B-2D).

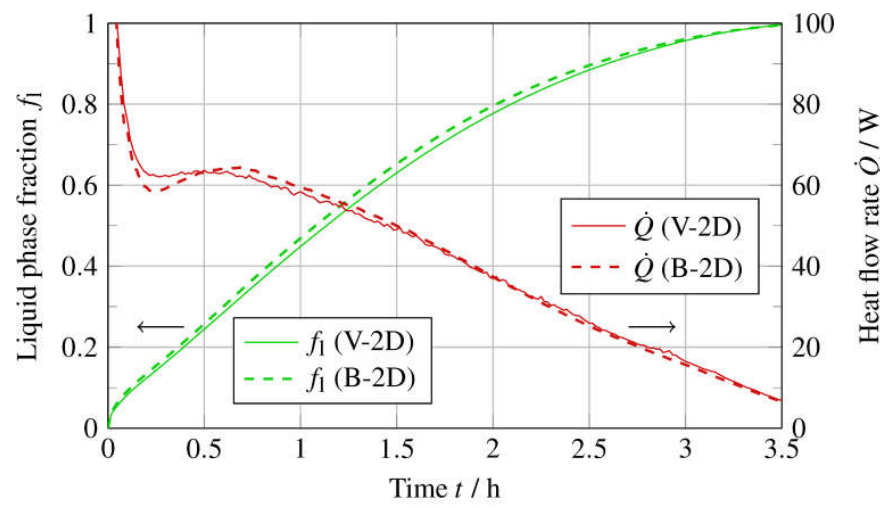

Figure 7: Comparison of liquid phase fraction of the PCM and heat flow rate into the PCM plotted over time for the VOF model (V-2D) and the Boussinesq model (B-2D).

The resulting curves of both models are qualitatively similar and there are only small deviations. The quantitative difference in liquid phase fraction has a mean value of $1.4 \%$ and a maximum value of $2.2 \%$. The heat flow rates deviate by $3.1 \%$ in the mean with a maximum difference of $10.1 \%$ that occurs only over a small period of time. Although the shape of the solid phase was observed to be slightly different in the last section, the integral phase state is nearly identical in both models. Conclusively, despite the differences in the models and observed qualitative differences, the overall melting process is similar.

\subsubsection{Velocity extrema}

The velocities are also analyzed over time. However, it is not straight forward to find a single velocity value that is representative for the melting process. If we would evaluate the velocity at a certain fixed point or even multiple points, the choice of position of this point would be critical for the results. Either the same velocity would be obtained for a long period of time or the velocity could be zero the majority of the time. The mean value of the velocity magnitude is also not meaningful, because the velocities outside of the narrow boundary layers are close to zero, which would lead to a small mean value. The solution was to calculate the velocity extrema separately for the $x$ - and $y$-direction and for many evaluation time steps. The result is shown in Figure 8.

The velocities $v$ in $y$-direction are very similar to each other, while the velocities $u$ in $x$-direction show a qualitative difference at least at the beginning of the melting process. The mean deviation of velocities $u$ in $x$-direction is $20 \%$ and the mean deviation of velocities $v$ in $y$-direction is $8.2 \%$. At the beginning of melting in the VOF model (V-2D), the velocities $\max (u)$ in positive $x$-direction decrease earlier and the velocities $\min (u)$ in negative $x$-direction remain higher. The differences in $u$ are probably due to the different boundary condition at the top. Overall, the differences in the velocity extrema are not substantial and probably negligible.
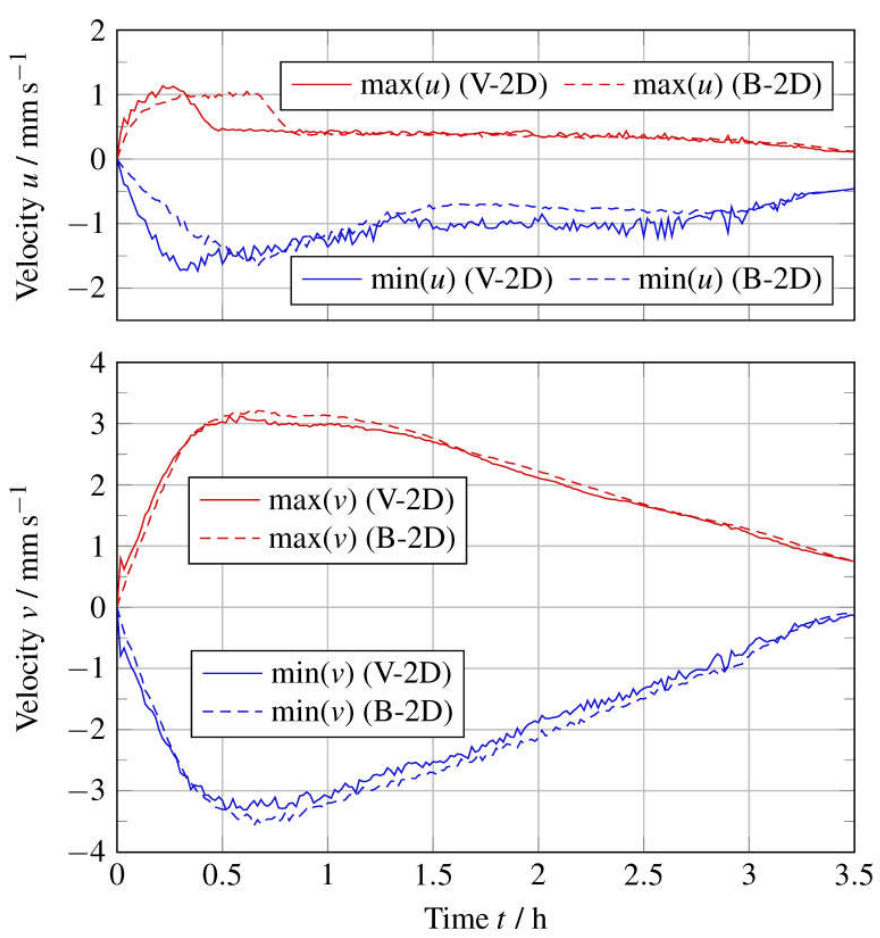

Figure 8: Comparison of velocity extrema in $x$ - and $y$-direction plotted over time for the VOF (V-2D) and the Boussinesq model (B2D).

\subsubsection{Temperatures}

The temperatures were evaluated at the three distinct positions $T_{\mathrm{PCM}, 1}, T_{\mathrm{PCM}, 2}$ and $T_{\mathrm{PCM}, 3}$ shown in Figure 2 and given in Table 1. The simulated results for the VOF model (V-2D) and the Boussinesq model (B-2D) are plotted over time in Figure 9.

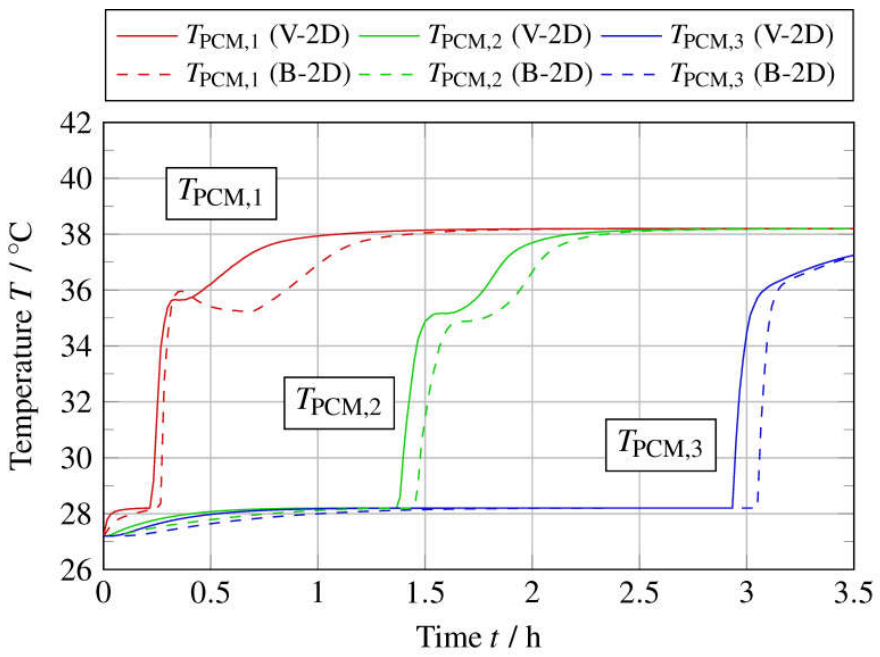

Figure 9: Comparison of Temperatures at three positions over time for the VOF (V-2D) and the Boussinesq model (B-2D).

The curves for temperature $T_{\mathrm{PCM}, 1}$ have a qualitative difference at the time right after the melting front passed the temperature probe position after $t=0.5 \mathrm{~h}$. However, the curves for the temperature $T_{\mathrm{PCM}, 2}$ and temperature $T_{\mathrm{PCM}, 3}$ are 
similar and only shifted in time. The mean difference over the melting process is $1.1 \%$ for $T_{\mathrm{PCM}, 1}, 1.2 \%$ for $T_{\mathrm{PCM}, 2}$ and $1.0 \%$ for $T_{\mathrm{PCM}, 3}$. Hence, the differences in temperature are mostly negligible.

\subsection{Comparison of the $3 D$ Boussinesq model (B-3D) with the experiment}

The comparison of the two different models in 2D showed only minor differences. In conclusion, the Boussinesq approximation, the neglected volume expansion and the assumption of constant material properties are valid for this test case. Hence, we preferred the simpler Boussinesq model to simulate the $3 \mathrm{D}$ case. The $3 \mathrm{D}$ case additionally included viscous boundary layer effects at the front and back enclosure walls. We also adjusted the thermal boundary conditions at all sides to data from the experiment to include the small but remaining heat flows over the boundaries. This way, the simulation model could be precisely compared to the experiment. As in the section before, the primary quantities of interest are the phase state, the velocity and the temperature. In a first step, the 2D fields of phase state and velocities in the liquid phase in the mid-plane $(z=25 \mathrm{~mm})$ of the enclosure are evaluated in the simulation and compared to experimental results [3]. In a second step, the quantitative values of liquid phase fraction, velocity extrema and temperatures in the PCM are evaluated from simulated data and compared with experimental results [3].

\subsection{1. $2 D$ fields of phase state and velocity}

As in the last section, the fields of liquid phase fraction and velocities by natural convection in the liquid phase were evaluated. But this time, data from the B-3D model were compared to the validation experiment. The results are illustrated in Figure 10 for the time $t=3600 \mathrm{~s}$. The solid phase of the PCM is drawn in black as are the solid walls. In the liquid phase, filled contours show the velocity magnitude and vectors show the velocity direction as well as the magnitude. In the simulation model B-3D shown on the left side, the velocity is evaluated in a plane in the middle of the enclosure $(z=25 \mathrm{~mm})$. The data looks slightly disturbed at the phase front, which is mostly due to a coarser grid in the 3D case compared to the $2 \mathrm{D}$ case and the interpolation from control volume centered values onto the faces of the mid-plane.

The experimental data was obtained with a shadowgraph technique to obtain the phase state and a particle image velocimetry technique to measure velocities in a plane in the middle of the enclosure $(z=25 \mathrm{~mm})$, see Vogel and Bauer [3].

The solid phase has a smaller and narrower appearance in the simulation compared to the experiment. While the velocity magnitudes are similar at the phase boundary of the PCM, the simulated velocities exceed the measured velocities at the heated wall. Since the flow is laminar, see section 3, turbulence should not be the reason for deviations. A detailed 3D model with VOF method including volume expansion and variable material properties would probably lead to a slightly better qualitative agreement than the simplified Boussinesq model (B-3D) used here, but the computational effort would be significantly higher. And there are certain other features in the experiment, which would still not be modeled in the simulation, e.g. gas bubbles rising from the solid phase. Conclusively, regarding the simplifications in this model, the simulation qualitatively agrees with the experiment. The quantitative evaluation follows in the next sections. 


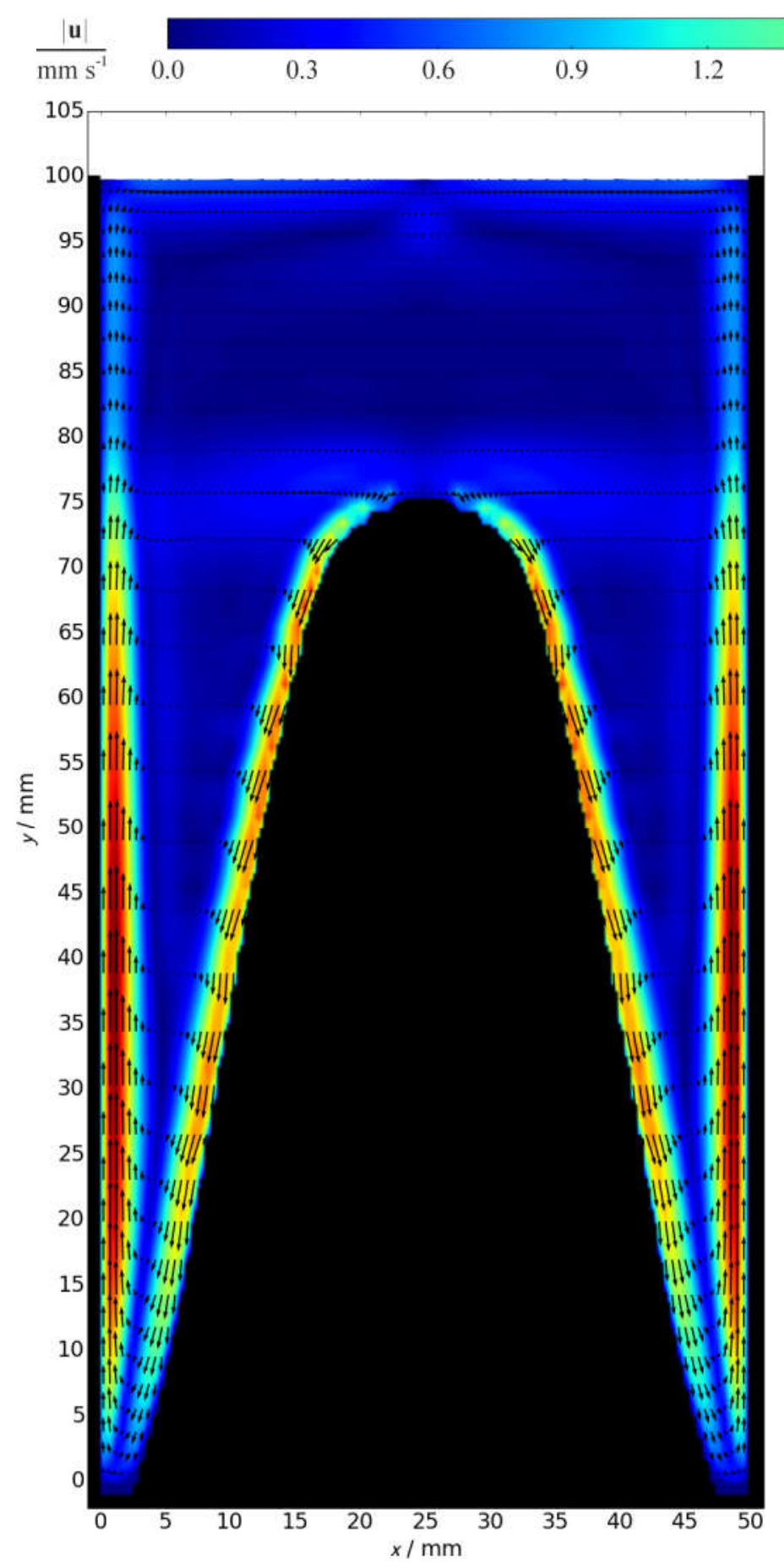

Simulation (B-3D) (midplane)

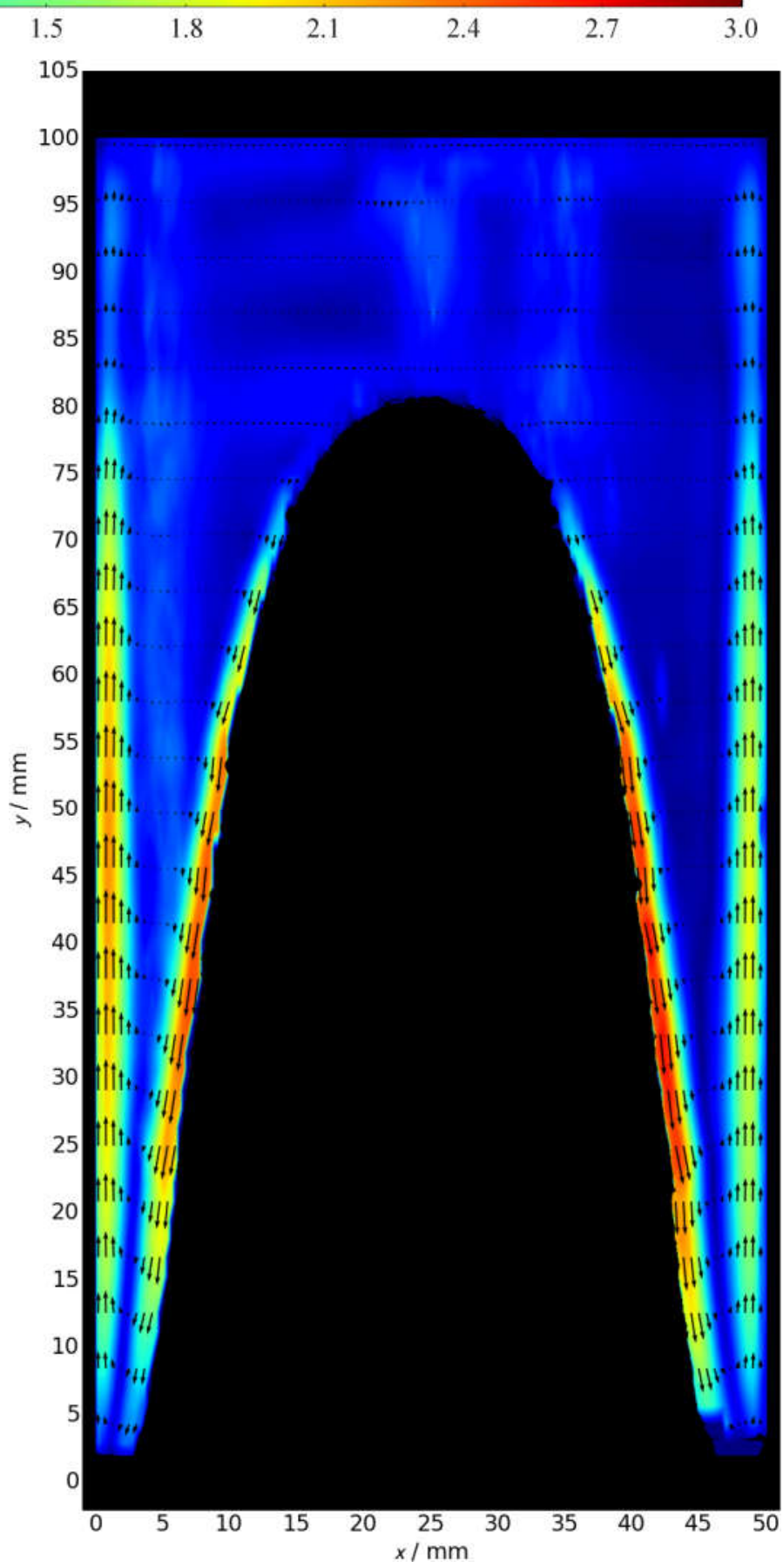

Experiment (midplane)

Figure 10: Comparison of simulation results with the 3D Boussinesq model (B-3D) including boundary effects (left side) with results from the validation experiment [3] (right side) at time $t=3600 \mathrm{~s}\left(R a_{H}=2.0 \cdot 10^{8}\right.$, Ste $\left.=0.092, \operatorname{Pr}=52.2, A=4\right)$. 


\subsubsection{Liquid phase fraction}

We evaluated the liquid phase fraction in the simulation with the B-3D model and compared it to the experimentally measured data [3], which includes error bars for systematic and statistic measurement uncertainties. The results are shown in Figure 11.

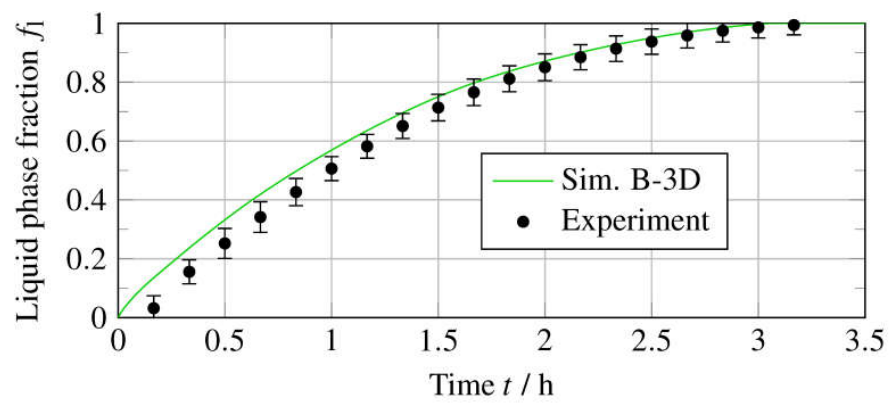

Figure 11: Comparison of liquid phase fraction over time from the 3D simulation (B-3D) and from experimentally measured data [3].

A maximum deviation of the simulation from the experiment of $10 \%$ is found at the beginning of the melting process. This might be due to the higher thermal inertia of the experimental system. Although the experimentally measured boundary temperatures were used in the boundary conditions of the simulation, the few measurement positions cannot completely capture the temperature variation at the boundary of the test cell. However, since the mean deviation is only $4 \%$, the overall melting process is found to be similar in the simulation and the experiment.

\subsubsection{Velocity extrema}

The velocities from the simulations with the B-3D model were also compared to the experiment, where velocities were measured by particle image velocimetry (PIV) in a middle $x-y$ plane [3]. The velocity extrema were again calculated in both axis directions. The error bars include systematic and statistic measurement uncertainties. The results are shown in Figure 12.

The maximum velocity magnitudes are mostly higher in the simulation compared to the experiment. The simulated velocities $u$ are slightly higher in both negative (left) and positive (right) $x$-direction. The simulated velocities $v$ are significantly higher in the positive (upward) $y$-direction in the wall boundary layer but similar in the negative (downward) $y$ direction in the phase front boundary layer. The mean deviation is about $60 \%$ for $u$ and $20 \%$ for $v$.

A possible explanation for lower velocities in the experiment could be attributed to non-uniform temperatures at the heater wall that were not captured by our measurement positions, for example a horizontal temperature gradient. Another possible reason is the obstruction of fluid flow by the thermocouples despite their small diameter. On the other hand, the simulation might not have captured the wall boundary layer correctly due to turbulence effects that we did not include since the flow should be laminar according to Equation (3) by Elder [33].
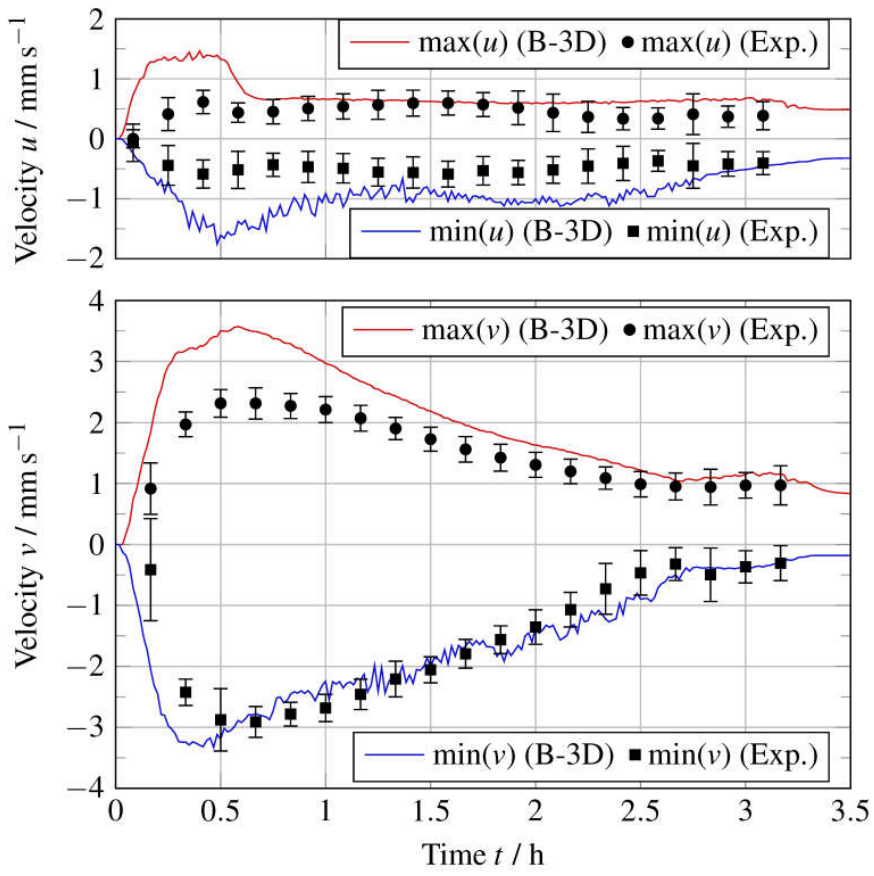

Figure 12: Comparison of $u$ - and $v$-velocity extrema over time from the simulation model B-3D and from PIV-measurements in the experiment [3].

\subsubsection{Temperatures}

Finally, temperatures evaluated in the simulation with the B-3D model are compared to temperatures measured with thermocouples in the experiment [3] in Figure 13. The temperature probe positions are shown in Figure 2 and their coordinates are given in Table 1. The error bars include systematic and statistic measurement uncertainties, which can become quite large at steep gradients, because these cannot be exactly reproduced in repeated measurements.

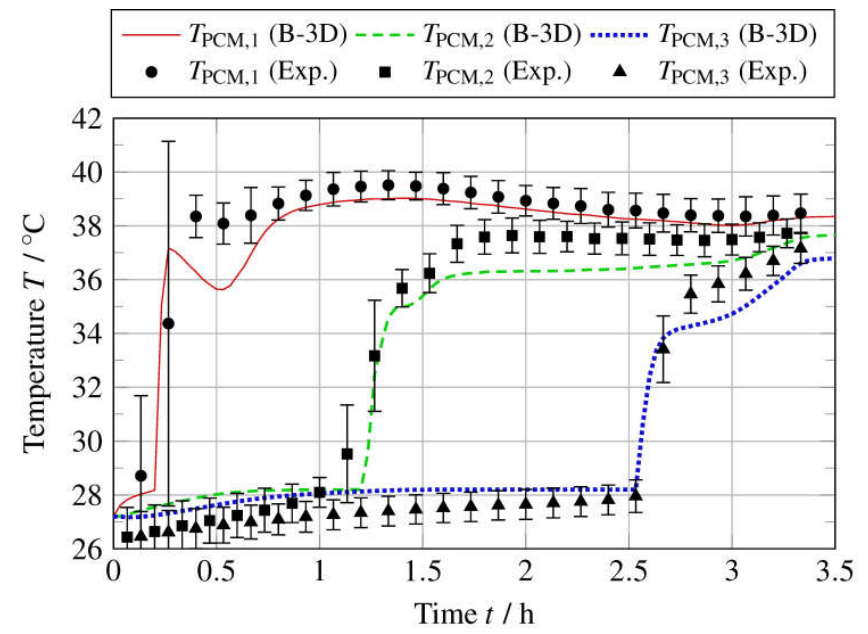

Figure 13: Comparison of temperatures at three positions over time from the evaluation probes in the simulation with the B-3D model and from the thermocouple measurements in the experiment [3].

The simulated temperature curves are similar to the experimentally measured data. The instants of melting are captured well. However, the temperature $T_{\mathrm{PCM}, 1}$ has a different curve around the time $t \approx 0.5 \mathrm{~h}$, but agrees well for the remaining time. The temperatures $T_{\mathrm{PCM}, 2}$ and $T_{\mathrm{PCM}, 3}$ agree well 
initially, but they are slightly lower after phase change is finished in the vicinity of the probe at times $t>1.6 \mathrm{~h}$ and $t>2.7 \mathrm{~h}$, respectively. The mean deviations over the melting process are $1.8 \%$ for $T_{\mathrm{PCM}, 1}$ and $2.8 \%$ for both $T_{\mathrm{PCM}, 2}$ and $T_{\mathrm{PCM}, 3}$.

\section{Conclusions}

The comparison of the detailed modeling approach with variable material properties and the VOF-method (V-2D) to the simplified approach with constant material properties and the Boussinesq approximation (B-2D) revealed differences only upon closer inspection. Deviations are seen mostly in the velocities and temperatures. However, the liquid phase fraction and heat flow rate, which indicate the overall charging state and power of a storage system, are very similar. In conclusion, while the simplified model (B-2D) is not able to reproduce the melting process in every detail, it is sufficiently accurate to be used in the design process of latent thermal energy storage systems.

The comparison of the simplified model in three dimensions with boundary effects (B-3D) to the validation experiment showed that the simulated phase state and velocities are qualitatively similar to the validation experiment. Only the simulated mean velocity extrema are $60 \%$ higher for the velocity $u$ in $x$-direction and $20 \%$ higher for the velocity $v$ in $y$-direction compared to the experiment and it is not yet understood what exactly is the reason for this. However, the liquid phase fraction only deviates by a mean value of $4 \%$ and temperatures by a mean value of $2.8 \%$ in the worst case. These results indicate that the simplified model (B-3D) is accurate enough to reproduce the experimentally measured melting process and that it is qualified for further studies to analyze the impact of natural convection in LTES systems.

Since only one set of parameters was investigated in this case study, the next step would be to do a parameter study to find the boundary of validity of the Boussinesq approximation in the simplified model. For larger subcooling or larger driving temperature differences, we would expect that the simplified model will suffer from low accuracy and only the detailed model with VOF method will be accurate enough.

In this work, we studied a low temperature material for minimum boundary effects and accurate experimental results, which is advantageous for a validation study. However, it is not known if the results with high-temperature materials would be similar or if there will be other significant influences such as radiation heat transfer that are not yet included in the simulation model. Hence, an extension of this study to high temperature phase change materials is advised.

\section{Acknowledgments}

The authors are grateful to Dan Bauer and Stefan Zunft for insightful discussions.

\section{References}

[1] Mehling H, Cabeza LF. Heat and cold storage with PCM. Springer-Verlag Berlin Heidelberg; 2008. doi:10.1007/978-3540-68557-9.

[2] Bauer T, Steinmann W-D, Laing D, Tamme R. Thermal Energy Storage Materials and Systems. Annu. Rev. Heat Transf., 2012, p. 131-77.

doi:10.1615/AnnualRevHeatTransfer.2012004651.

[3] Vogel J, Bauer D. Phase state and velocity measurements with high temporal and spatial resolution during melting of $n$ octadecane in a rectangular enclosure with two heated vertical sides. Int J Heat Mass Transf 2018;127:1264-76. doi:10.1016/j.ijheatmasstransfer.2018.06.084.

[4] Voller VR, Cross M, Markatos NC. An enthalpy method for convection/diffusion phase change. Int J Numer Methods Eng 1987;24:271-84. doi:10.1002/nme.1620240119.

[5] Salcudean M, Abdullah Z. On the Numerical Modelling of Heat Transfer during Solidification Processes. Int J Numer Methods Eng 1988;25:445-73. doi:10.1002/nme.1620250212.

[6] Samarskii AA, Vabishchevich PN, Iliev OP, Churbanov AG. Numerical simulation of convection/diffusion phase change problems-a review. Int J Heat Mass Transf 1993;36:4095106. doi:10.1016/0017-9310(93)90071-D.

[7] Voller VR. An Overview of Numerical Methods for Solving Phase Change Problems. In: Minkowycz WJ, Sparrow EM, editors. Adv. Numer. HEAT Transf. Vol. 1, Taylor \& Francis; 1997, p. 341-80.

[8] Hu H, Argyropoulos SA. Mathematical modelling of solidification and melting: a review. Model Simul Mater Sci Eng 1999;4:371-96. doi:10.1088/0965-0393/4/4/004.

[9] Dutil Y, Rousse DR, Salah N Ben, Lassue S, Zalewski L. A review on phase-change materials: Mathematical modeling and simulations. Renew Sustain Energy Rev 2011;15:11230. doi:10.1016/j.rser.2010.06.011.

[10] Dhaidan NS, Khodadadi JM. Melting and convection of phase change materials in different shape containers: A review. Renew Sustain Energy Rev 2015;43:449-77. doi:10.1016/j.rser.2014.11.017.

[11] Lacroix M, Voller VR. Finite Difference solutions of solidification phase change problems: transformed versus fixed grids. Numer Heat Transf Part B Fundam An Int J Comput Methodol 1990;17:25-41. doi:10.1080/10407799008961731.

[12] Viswanath R, Jaluria Y. A comparison of different solution methodologies for melting and solidification problems in enclosures. Numer Heat Transf Part B Fundam 1993;24:77105. doi:10.1080/10407799308955883.

[13] Voller V, Prakash C. A fixed grid numerical modelling methodology for convection-diffusion mushy region phasechange problems. Int J Heat Mass Transf 1987;30:1709-19. doi:10.1016/0017-9310(87)90317-6.

[14] Brent AD, Voller VR, Reid KJ. Enthalpy-Porosity Technique for Modeling Convection-Diffusion Phase Change: Application to the Melting of a Pure Metal. Numer Heat Transf 1988;13:297-318. doi:10.1080/10407788808913615.

[15] Voller VR, Swaminathan CR. General Source-Based Method for Solidification Phase Change. Numer Heat Transf Part B 1991;19:175-89. doi:10.1080/10407799108944962.

[16] Patankar S V, Spalding DB. A calculation procedure for heat, mass and momentum transfer in three-dimensional parabolic flows. Int J Heat Mass Transf 1972;15:1787-806. doi:10.1016/0017-9310(72)90054-3.

[17] Chorin AJ. Numerical solution of the Navier-Stokes equations. Math Comput 1968;22:745-62. doi: $10.2307 / 2004575$. 
[18] Galione PA, Lehmkuhl O, Rigola J, Oliva A. Fixed-grid modeling of solid-liquid phase change in unstructured meshes using explicit time schemes. Numer Heat Transf Part B 2014;65:27-52. doi:10.1080/10407790.2013.836399.

[19] Kozak Y, Ziskind G. Novel enthalpy method for modeling of PCM melting accompanied by sinking of the solid phase. Int J Heat Mass Transf 2017;112:568-86.

doi:10.1016/j.ijheatmasstransfer.2017.04.088.

[20] Hirt C., Nichols B. Volume of fluid (VOF) method for the dynamics of free boundaries. J Comput Phys 1981;39:20125. doi:10.1016/0021-9991(81)90145-5.

[21] Shatikian V, Ziskind G, Letan R. Numerical investigation of a PCM-based heat sink with internal fins. Int J Heat Mass Transf 2005;48:3689-706.

doi:10.1016/j.ijheatmasstransfer.2004.10.042.

[22] Shmueli H, Ziskind G, Letan R. Melting in a vertical cylindrical tube: Numerical investigation and comparison with experiments. Int J Heat Mass Transf 2010;53:4082-91. doi:10.1016/j.ijheatmasstransfer.2010.05.028.

[23] Hosseinizadeh SF, Tan FL, Moosania SM. Experimental and numerical studies on performance of PCM-based heat sink with different configurations of internal fins. Appl Therm Eng 2011;31:3827-38.

doi:10.1016/j.applthermaleng.2011.07.031.

[24] Hannoun N, Alexiades V, Mai TZ. Resolving the Controversy over Tin and Gallium Melting in a Rectangular Cavity Heated from the Side. Numer Heat Transf Part B 2003;44:253-76. doi:10.1080/10407790390226974.

[25] Kadri S, Dhifaoui B, Dutil Y, Jabrallah S Ben, Rousse DR. Large-Scale Experimental Study of a Phase Change Material: Shape Identification for the Solid-Liquid Interface. Int J Thermophys 2015;36:2897-915. doi:10.1007/s10765-0151935-y.

[26] Bertrand O, Binet B, Combeau H, Couturier S, Delannoy Y, Gobin D, et al. Melting driven by natural convection A comparison exercise: first results. Int J Therm Sci 1999;38:526. doi:10.1016/S0035-3159(99)80013-0.

[27] Gobin D, Le Quéré P. Melting from an isothermal vertical wall. Synthesis of a numerical comparison exercise. Comput Assist Methods Eng Sci 2000;7:289-288.

[28] Campbell TA, Koster JN. Visualization of liquid-solid interface morphologies in gallium subject to natural convection. J Cryst Growth 1994;140:414-25. doi:10.1016/0022-0248(94)90318-2.

[29] Gau C, Viskanta R. Melting and Solidification of a Pure Metal on a Vertical Wall. J Heat Transfer 1986;108:174. doi:10.1115/1.3246884.

[30] Elder JW. Laminar free convection in a vertical slot. J Fluid Mech 1965;23:77. doi:10.1017/S0022112065001246.

[31] Jany P, Bejan A. Scaling theory of melting with natural convection in an enclosure. Int J Heat Mass Transf 1988;31:1221-35. doi:10.1016/0017-9310(88)90065-8.

[32] Batchelor GK. Heat transfer by free convection across a closed cavity between vertical boundaries at different temperatures. Q Appl Math 1954;12:209-33.

[33] Elder J. Turbulent free convection in a vertical slot. J Fluid Mech 1965;23:99-111.

[34] Galione PA, Lehmkuhl O, Rigola J, Oliva A. Fixed-grid numerical modeling of melting and solidification using variable thermo-physical properties - Application to the melting of n-Octadecane inside a spherical capsule. Int J Heat Mass Transf 2015;86:721-43. doi:10.1016/j.ijheatmasstransfer.2015.03.033.

[35] Inc A. ANSYS 15 Fluent Theory Guide 2015.

[36] Carman PC. Fluid flow through granular beds. Chem Eng Res Des 1997;75:S32-48. doi:10.1016/S02638762(97)80003-2.
[37] VDI Heat Atlas (VDI Wärmeatlas). Berlin, Heidelberg: Springer Vieweg; 2013. doi:10.1007/978-3-642-19981-3.

[38] Vogel J, Felbinger J, Johnson M. Natural convection in high temperature flat plate latent heat thermal energy storage systems. Appl Energy 2016;184:184-96. doi:10.1016/j.apenergy.2016.10.001.

[39] Versteeg HK, Malalasekera W. An introduction to computational fluid dynamics: The Finite Volume Method. 2nd ed. 2007.

[40] ANSYS Inc. ANSYS 15 Fluent User's Guide 2015. 